\title{
Bright split red fluorescent proteins with enhanced complementation efficiency for the tagging of endogenous proteins and visualization of synapses
}

Siyu Feng ${ }^{1}$, Aruna Varshney², Doris Coto Villa², Cyrus Modavi ${ }^{3}$, John Kohler ${ }^{4}$, Fatima Farah², Nebat Ali ${ }^{2}$, Joachim Dieter Mueller ${ }^{4}$, Miri VanHoven², Bo Huang ${ }^{5,6,7, *}$

${ }^{1}$ The UC Berkeley-UCSF Graduate Program in Bioengineering, San Francisco, CA 94143 USA;

2 Department of Biological Sciences, San Jose State University, San Jose, CA 95192 USA;

${ }^{3}$ Department of Bioengineering and Therapeutic Sciences, University of California in San

Francisco, San Francisco, CA 94143, USA;

${ }^{4}$ School of Physics and Astronomy, University of Minnesota, Minneapolis, MN 55455, USA;

${ }^{5}$ Department of Pharmaceutical Chemistry, University of California in San Francisco, San

Francisco, CA 94143, USA;

${ }^{6}$ Department Biochemistry and Biophysics, University of California, San Francisco, San

Francisco, CA 94143, USA;

${ }^{7}$ Chan Zuckerberg Biohub, San Francisco, CA 94158, USA.

*Correspondence should be addressed to bo.huang@ucsf.edu 


\begin{abstract}
Self-associating split fluorescent proteins (FPs) have been widely used for labeling proteins, scaffolding protein assembly and detecting cell-cell contacts. Newly developed self-associating split FPs, however, have suffered from suboptimal fluorescence signal. Here, by investigating the complementation process, we have demonstrated two approaches to improve split FPs: assistance through SpyTag/SpyCatcher interaction and directed evolution. The latter has yielded two split sfCherry3 variants with substantially enhanced overall brightness, facilitating the tagging of endogenous proteins by gene editing. Based on sfCherry3, we have further developed a new red-colored trans-synaptic marker called Neuroligin-1 sfCherry3 Linker Across Synaptic Partners (NLG-1 CLASP) for multiplexed visualization of neuronal synapses in living animals, demonstrating its broad applications.
\end{abstract}




\section{Introduction}

Self-associating split fluorescent proteins (FPs) are a powerful tool for protein labeling and livecell imaging. In this system, the eleventh $\beta$-strand of FP (FP 11,16 amino acids) is separated out from the remainder of FP ( $\left.\mathrm{FP}_{1-10}\right)$ and is genetically fused to the protein of interest (POI). Specific fluorescence signal is detected when $\mathrm{FP}_{1-10}$ reconstitutes with the on-target $\mathrm{FP}_{11}$ to generate a functional fluorescent protein. Since the initial development of self-associating split GFP $_{1-10 / 11}(1)$, this technology has been modified and adapted for an extensive range of applications including protein labeling and visualization (2-4), scaffolding protein assembly (3), protein solubility and aggregation assays $(5,6)$, and monitoring the membrane fusion process (7). One prominent application is generating a library of human cells with fluorescently tagged endogenous proteins via CRISPR/Cas9-mediated homology-directed repair (4). The small size of the GFP 11 tag markedly improves the knock-in efficiency and simplifies the donor DNA preparation. In another application, the split GFP 1 -10/11 system has also been utilized to visualize synapses in living nervous systems by Neuroligin-1 GFP Reconstitution Across Synaptic Partners (NLG-1 GRASP) (8).

While all these applications have focused on a single, green-colored channel, expanding the color palette will greatly benefit the investigation of more complex biological systems by enabling multicolor imaging. For this purpose, we have recently developed a red-colored split sfCherry 1 -10/11 (3) and then a brighter split sfCherry21-10/11 (9), enabling dual-color endogenous labeling in human cells using orthogonal $\mathrm{FP}_{11}$ tags (9) and visualization of Listeria protein secretion in infection (10). However, unlike split GFP $1-10 / 11$, which is as bright as its full-length, split sfCherry2 $2_{1-10 / 11}$ produces substantially lower overall fluorescence signal than its full-length counterpart.

Here, we have characterized the complementation mechanism of split FP systems by examining their overall and single-molecule fluorescence brightness. The results suggest a 2-step complementation model in which the affinity between the $\mathrm{FP}_{1-10}$ and $\mathrm{FP}_{11}$ fragment is the major limitation to the overall fluorescence signal. Based on this model, we have devised a SpyTag/SpyCatcher-assisted approach to improve the complementation efficiency of sfCherry $2_{1-10 / 11}$. Furthermore, we have engineered two split sfCherry3 variants with muchenhanced complementation efficiency through a combination of cycles of directed evolution and structure-based site-directed mutagenesis. For tagging endogenous proteins by gene editing, sfCherry3 improves the sorting efficiency for successfully knocked-in cells by 5-10 fold in six tested targets, as compared to sfCherry2. Moreover, we have also developed of a new red- 
colored trans-synaptic marker called Neuroligin-1 sfCherry3 Linker Across Synaptic Partners (NLG-1 CLASP). We established that like NLG-1 GRASP, NLG-1 CLASP labels connections between correct synaptic partners, and has a spatial pattern similar to that predicted by electron micrograph reconstruction $(11,12)$. As a validation, NLG-1 CLASP labeling is disrupted by loss of the clr-1 gene, which is required for synaptic partner recognition (13).

\section{Results}

\section{The complementation efficiency of split sfCherry2 and split mNeonGreen2 can be} explained by a dynamic association/dissociation equilibrium model.

Previously, we have shown that split GFP ${ }_{1-10 / 11}$ has nearly identical overall brightness as its fulllength counterpart, whereas both split mNeonGreen2 ${ }_{1-10 / 11}$ (mNG2 $\left.1-10 / 11\right)$ and split sfCherry2 ${ }_{1-10 / 11}$ are substantially dimmer (9). This sub-optimal performance of $\mathrm{mNG}_{1-10 / 11}$ and sfCherry2 $2_{1-10 / 11}$ could be attributed to either (a) the lower molecular brightness of complemented split FPs or (b) incomplete complementation between the $\mathrm{FP}_{1-10}$ and $\mathrm{FP}_{11}$ fragments. To test the first possibility, we measured the single-molecule brightness of these three split FPs and their full-length counterparts in living cells using fluorescence fluctuation spectroscopy (14). We observed no significant difference in single-molecule brightness between the split and the full-length FPs in all three cases (Fig. 1A). Therefore, incomplete complementation should be the cause of the reduced overall fluorescence signal. The ratio of overall fluorescence between split and fulllength FPs then reflects the complementation efficiency.

For $m N G 2_{1-10 / 11}$ and sfCherry2 ${ }_{1-10 / 11}$, to determine their complementation efficiency and compare them to GFP 1-10/11, we took a similar approach as previously done (9). We transiently expressed in HEK 293T cells the full-length FP or the two fragments: FP $_{1-10}$ and FP $_{11}$ on a well-folded carrier protein. We quantified whole cell fluorescence by flow cytometry while using a coexpressed infrared fluorescent protein mIFP to measure the expression level. mIFP was linked to the full-length FP or the $\mathrm{FP}_{11}$ fragment through a P2A self-cleavage site to ensure equimolar expression. Fluorescence intensities in infrared and green/red channels of each single cell events were displayed in log-log scale scatter plots (Figs. 1B-D).

In the cases of full-length FPs, as expected, single cell fluorescence intensities followed the trend line with a slope of 1 because mIFP was expressed equimolarly (Fig. 1E). In the split cases, split GFP 1-10/11 almost completely followed the same diagonal line as its full-length 
counterpart (except for a subtle deviation at the lower-expression end) (Fig. 1B), suggesting a complementation efficiency of almost $100 \%$ across a wide range of expression levels. In contrast, both split mNG2 ${ }_{1-10 / 11}$ and split sfCherry2 ${ }_{1-10 / 11}$ deviated from the trend lines of their full length counterparts (Figs. $1 C$ \& D). This observation prompted us to consider a 2-step complementation process for self-associating split FPs: the two fragments undergo a reversible association/dissociation equilibrium before entering an irreversible process (fig. S1) of folding and/or chromophore maturation:

$$
\mathrm{FP}_{1-10}+\mathrm{FP}_{11} \rightleftharpoons \mathrm{FP}_{1-10 / 11}^{*} \stackrel{\text { folding/maturation }}{\longrightarrow} \mathrm{FP}_{1-10 / 11} \rightarrow \text { degradation }
$$

We have verified that in our co-transfection scheme, the expression levels (concentrations) of $\mathrm{FP}_{1-10}$ and $\mathrm{FP}_{11}$ fragments are proportional through an independent experiment in cells expressing mIFP-P2A-sfCherry2 ${ }_{11}$-Carrier and TagBFP-P2A-sfCherry2 ${ }_{1-10}$ (Fig. 1E). Then, at a steady state, this 2-step model predicts the following relationship between the two channels of flow cytometry (see Supplementary Notes):

$$
[\mathrm{mIFP}] \propto \sqrt{K_{D}{ }^{\prime}\left[\mathrm{FP}_{1-10 / 11}\right]}+\left[\mathrm{FP}_{1-10 / 11}\right]
$$

where [mIFP] represents the sum of concentrations of uncomplemented and complemented $\mathrm{FP}_{11},\left[\mathrm{FP}_{1-10 / 11}\right]$ is the concentration of matured $\mathrm{FP}_{1-10 / 11}$, and $K_{D}{ }^{\prime}$ is the effective dissociation constant of the overall complementation/maturation process. When $K_{D}{ }^{\prime}$ is much lower than the expression level of $\mathrm{FP}_{11}$ (the case of $\mathrm{GFP}_{1-10 / 11}$ ), the second term dominates, leading to a near proportional relationship between the two flow cytometry channels (slope of 1 in log-log plot, Fig. 1B). In the opposite case of high $K_{D}{ }^{\prime}$ (the cases of $m N G 2_{1-10 / 11}$ and sfCherry2 $1-10 / 11$ ), the first term dominates, resulting in log-log scatter plots following more closely to slope-2 trend lines, matching our observations (Figs. 1C\&D).

\section{SpyTag/SpyCatcher interaction improves complementation of split sfCherry2.}

Our model indicates that the complementation efficiency of split mNG2 $2_{1-10 / 11}$ and sfCherry $2_{1-10 / 11}$ improves with raised local concentration of fragments, leading to enhanced overall fluorescence signal. Therefore, we sought to utilize a pair of high-affinity binding partners to bring the two fragments into spatial proximity. Because a major advantage of the split $\mathrm{FP}_{1-10 / 11}$ is to label endogenous proteins through knocking-in the short $\mathrm{FP}_{11}$ peptide, it is preferable to have a small binding partner for the $\mathrm{FP}_{11}$ fragment. For this purpose, we chose the SpyTag/SpyCatcher (15) system, a peptide-protein pair that undergoes irreversible binding through formation of an 
isopeptide bond. The 13-amino-acid (aa) SpyTag is sufficiently short that even when concatenated with GFP $_{11}$, the resulting sequence remains small enough for knock-in using synthetic oligo donor DNAs (16).

We examined SpyTag/SpyCatcher-assisted complementation on sfCherry2 $2_{1-10 / 11}$ using the recently improved Spy002 pair (17) (Fig. 2A Upper panel). We fused SpyCatcher to the Nterminus of sfCherry2 ${ }_{1-10}$ (the $\mathrm{C}$-terminus is the split site) through a flexible linker in either 6 aa or 15 aa length (Fig. 2A Lower panel). We generated concatenated tags with SpyTag on either the $\mathrm{N}$ - or C-terminus of sfCherry 211 with a double-glycine linker. We performed similar flow cytometry experiments as described earlier except that mIFP was replaced by TagBFP. Among the four possible combinations of binders/tags (fig. S2), SpyCatcher-6aa-sfCherry2 ${ }_{1-10}$ with SpyTag-sfCherry2 11 -TagBFP demonstrated the most pronounced shift towards a trend line with a slope of 1 in the scatter plot (Fig. 2B). We have further verified that a shorter SpyTagsfCherry $2{ }_{11}$ fusion without the double-gylcine spacer behaved as efficiently (data not shown).

We validated the improvement in overall brightness for cellular microscopy by labeling the $\mathrm{N}$ terminus of histone $2 \mathrm{~B}(\mathrm{H} 2 \mathrm{~B})$ with SpyTag-sfCherry2 ${ }_{11}$-TagBFP and co-expressing it with either sfCherry $2_{1-10}$ or SpyCatcher-6aa-sfCherry2 ${ }_{1-10}$ fusion in HEK 293T cells. We observed the Spyassisted system (Fig. 2C) could mark the nuclei with much stronger fluorescence signal with the same expression vectors.

\section{Engineering split sfCherry3 for better complementation efficiency}

Previously, we engineered sfCherry2 ${ }_{1-10 / 11}$ using a spacer-insertion strategy (9). This strategy was based on inserting a 32-aa spacer between the sfCherry ${ }_{1-10}$ and sfCherry ${ }_{11}$ coding regions. Beyond allowing mutagenesis of both fragments in a single PCR amplicon, the spatial constraints imposed by the linker were hypothesized to assist in the detection of the original mutations by raising the local concentrations of complimentary fragments. To increase the screening stringency for complementation-enhancing mutations, we chose to express the fragments of sfCherry2 as separate proteins using a pETDuet vector (Fig. 3A). Considering short peptides are prone to degradation in E. coli, we fused the sfCherry $2_{11}$ sequence to the $\mathrm{N}$ terminus of a well-folding carrier protein (SpyCatcher in our case).

We subjected the sfCherry $2_{1-10}$ fragment to four rounds of error-prone PCR mutagenesis and screening. In every round, a mixture of $\sim 20$ brightest variants were selected for the next round. 
The final isolated mutants were then subjected to one round of DNA shuffling. We have not mutated sfCherry $2_{11}$ so that all variants still bind the identical sfCherry $2_{11}$ peptide. In the end, sfCherry3C with 5 substitutions, K45R, G52D, T106A, K182R, N194D (numbering starts from the $1^{\text {st }}$ Glu after the starting codon Met) was identified as the best variant after the directed evolution (Fig. 3B). All mutations were mapped to either surface orientated residues (T106A, $\mathrm{K} 182 \mathrm{R}$ ) or locations potentially interacting with the sfCherry2 ${ }_{11}$ peptide (K45R, G52D, and N194D).

To further improve the complementation efficiency of sfCherry3C, we introduced rational mutations inspired by a mCherry mutant named cp193g7 that is tolerant of circular permutations near our split-site (18), because this mutant contains multiple similar mutations as in sfCherry (19) and sfCherry2 (9). We combinatorially introduced the remaining mutations of cp193g7 (I7F, F65L, and L83W) into sfCherry3C through site-directed mutagenesis. Only the variant containing a single L83W mutation gave brighter signal than sfCherry3C, which we designated as sfCherry3V (Fig. 3C). Complemented sfCherry3 $\mathrm{C}_{1-10 / 11}$ has an identical emission spectrum as that of sfCherry2 (both split and full-length), whereas the emission spectrum of complemented sfCherry3 $\mathrm{V}_{1-10 / 11}$ is blue-shifted by $5 \mathrm{~nm}$ (Fig. 3D). Fluorescence fluctuation spectroscopy indicates that sfCherry $3 \mathrm{C}_{1-10 / 11}$ has the same single-molecule brightness as sfCherry2 (fig. S3). On the other hand, sfCherry3 $\mathrm{V}_{1-10 / 11}$ is dimmer at the single-molecule level (fig. S3), which might be attributed to a difference in two-photon excitation of the blue-shifted chromophore at 1000 $\mathrm{nm}$ in the fluorescence fluctuation spectroscopy measurement.

Next, we performed the flow cytometry analysis (Fig. 3E) on HEK 293T cells co-transfected with mIFP-P2A-sfCherry2 ${ }_{11}$-Carrier and sfCherry $X_{1-10}(X$ being $2,3 \mathrm{C}$ or $3 \mathrm{~V}$ ). By fitting the data points (excluding those below a threshold above the scattering background) to a line with a fixed slope of 2 (fig. S4, see Supplementary Notes), we found a substantial up-shift of the fitted lines from sfCherry2 to sfCherry3C and sfCherry3V. The shifts corresponded to increases in binding affinity by 2.5 -fold and 8.2 -fold, respectively, assuming the same single-molecule brightness under one-photon excitation for flow cytometry. This enhancement in binding affinity should lead to improved complementation efficiency (hence the overall brightness) at the same expression level of the $\mathrm{FP}_{1-10}$ fragment.

\section{Efficient endogenous protein labeling in human cells using sfCherry 3 variants.}


One unique application of $\mathrm{FP}_{11}$ tag is to generate library-scale fluorescently labelled endogenous proteins through genetic knock-in by homology-directed DNA repair. The small 16aa size of $\mathrm{FP}_{11}$ allows us to fit its DNA sequence and short homology arms ( 70 nt on either side) into commercially available 200 nt single-strand oligo-DNA (ssDNA). By electroporating Cas9/sgRNA ribonucleoprotein (RNP) and donor ssDNA into cells constitutively expressing the corresponding $\mathrm{FP}_{1-10}$ fragment, robust generation of FP-labeled human cell lines becomes fast and cost-effective (4). Multicolor knock-in has also been demonstrated by using orthogonal split FP systems to visualize differential distribution and interaction of multiple endoplasmic reticulum proteins (9).

The overall increased brightness of complemented sfCherry3 variants make them superior to sfCherry2 in the application of labeling endogenous proteins through knock-in. Because sfCherry3 and sfCherry2 share the same FP $_{11}$ fragment, we adopted a reversed strategy as our previously reported one: knock-in of sfCherry 211 into HEK 293 T wild-type cells through electroporation, followed by lentivirus infection for the three sfCherry ${ }_{1-10}$ variants (schematics in Fig. 4A). A total of six sfCherry $2_{11}$ cell lines were created, with knock-ins at: lamin A/C (LMNA, inner nuclear membrane), clathrin light chain A (CLTA), RAB11A, heterochromatin protein $1 \beta$ (HP1b), endoplasmic reticulum proteins SEC61b (translocon complex) and ARL6IP1 (tubular ER). We compared Fluorescence-Activated Cell Sorting (FACS) enrichment efficiency for each cell line after infection with lentivirus (fig. $4 B$ to $4 G$ ). In all examined targets, the sfCherry3 $\mathrm{C}_{1-10}$ and $\mathrm{sfCherry} 3 \mathrm{~V}_{1-10}$ groups displayed remarkable population enhancement in the redfluorescence-positive gate compared to sfCherry $2_{1-10}$, rendering the sorting process substantially faster. Practically, for targets like CLTA, SEC61b or ARL6IP1, we were able to gate the fluorescent population around the clear peak and have 5-10 fold higher yield of isolating cells with successful knock-in.

We further confirmed our knock-ins were on-target through confocal microscopy imaging (Fig. $4 B$ to $4 G$ ). Noticeably, sfCherry3 $V_{1-10}$ groups have a reduced tendency to show fluorescent puncta from lysosomes (Fig. 4G) which is reported in our previous work (9). This makes the sfCherry3 $\mathrm{V}_{1-10}$ the preferred protein fragment when labeling endogenous proteins involved in the endomembrane system.

\section{NLG-1 CLASP visualizes specific subsets of synapses in live animals.}


To visualize synapses between specific sets of pre- and postsynaptic neurons in live animals, the trans-synaptic marker NLG-1 GRASP was designed using split GFP fragments (8). Complementary split GFP $1-10 / 11$ fragments were linked via a flexible linker to the transmembrane synaptic protein Neuroligin, which localizes to both pre- and postsynaptic sites in C. elegans (8). When the two neurons in which the complementary pre- and postsynaptic markers are expressed form synapses, the split GFP fragments come into contact, reconstitute and fluoresce (Fig. 5A). Using NLG-1 GRASP, we discovered that the recognition between two synaptic partners, the PHB sensory neurons and the AVA interneurons, is mediated the secreted ligand UNC-6/Netrin, its canonical receptor UNC-40/Deleted in Colorectal Cancer (20), and the receptor protein tyrosine phosphatase (RPTP) CLR-1 (13). NLG-1 GRASP has also been adapted to many other systems, indicating that this technology is transferable (21). The addition of a red fluorescent trans-synaptic marker would greatly expand this system.

Leveraging the brightened split-sfCherry3 tagging system, we have developed the split sfCherry-based NLG-1 CLASP. The left and right PHB sensory neurons, located in the posterior of $C$. elegans, form the majority of their synapses with the left and right AVA and PVC interneurons $(11,12)$. In this study, we focused on synapses formed between the left and right PHB neurons with the left and right AVA interneurons. To visualize PHB-AVA synapses with this newly developed split sfCherry $3 \mathrm{C}$, constructs were generated in which the sequence encoding the large fragment sfCherry $3 \mathrm{C}_{1-10}$ was linked to the N-terminus of the neuroligin-1 (nlg-1) cDNA after the $n / g-1$ signal sequence via a flexible $12 \mathrm{GS}$ linker. This half of the marker was expressed in PHB neurons using a promoter that within the posterior half of the worm, is specific for these presynaptic neurons ( $\left.{ }_{g} g p a-6\right)$ (22). The complementary small fragment sfCherry3 $\mathrm{C}_{11}$ was similarly linked to $n / g-1$ and expressed in AVA neurons using a promoter that within the posterior half of the worm, is specific for these postsynaptic neurons ( $p f l p-18)$ (23). Transgenic lines carrying both ${ }_{p} g p a-6:: n / g-1:: s f C h e r r y 3 C_{1-10}$ and ${ }_{p} f l p-18:: n / g-1:: s f C h e r r y 3 C_{11}$ were generated. In wild-type animals, the distribution of red fluorescent puncta was similar to those observed in animals labeled with PHB-AVA NLG-1 GRASP (Fig. 5), and to that described by serial electron microscopy reconstruction $(11,12)$. To determine if these puncta were indeed synaptic, we introduced NLG-1 CLASP into clr-1/RPTP synaptogenesis mutants. In c/r-1/RPTP mutants, PHB-AVA NLG-1 GRASP fluorescence intensity is dramatically reduced, and a PHB circuit-specific behavior is disrupted, indicating a reduction in synaptogenesis between the two neurons (13). We found that NLG-1 CLASP fluorescence intensity was also dramatically 
reduced in clr-1/RPTP mutants (Fig. 5), further indicating that NLG-1 CLASP puncta are synaptic.

\section{Discussion}

Taken together, we have proposed a dynamic association/dissociation model to explain the complementation process of self-associating split FP system. We have performed whole cell fluorescence intensity measurement and single-molecule brightness quantification to validate this model. Inspired by our model, we have adopted the SpyTag/SpyCatcher system to enhance the local concentrations of fluorescent protein fragments, thereby improving its complementation efficiency and overall brightness. We have also designed an engineering strategy by expressing the two fragments individually through a pETDuet vector. Using this platform, we have developed a bright sfCherry $3 \mathrm{C}_{1-10 / 11}$ (and its variant sfCherry3 $\mathrm{V}_{1-10 / 11}$ ) with enhanced complementation efficiency, enabling the high-efficient generation of human cell lines with endogenously sfCherry-labeled proteins. Moreover, we have transformed the split sfCherry3C into a trans-synaptic marker called NLG-1 CLASP and have demonstrated the visualization of specific synapses in living animals.

The proposed 2-step model, consisting of a dynamic association/dissociation equilibrium followed by an irreversible folding/maturation process, can be generalized to other split fluorescent proteins, including the non-self-associating ones used to monitor protein-protein interaction in bimolecular fluorescence complementation (BiFC) assays (24) (25). In fact, there is no definitive boundary between the non-self-associating and self-associating split FPs. Instead, their only difference is in the spontaneous binding affinity, which can be characterized by $K_{D}{ }^{\prime}$ in our model. For BiFC analysis, this affinity is one of the major determinant of the sensitivity. If it is too low, the split FP will fail to produce sufficient complementation signal even when the probed molecular interaction does occur. On the other hand, the opposite extreme leads to high background complementation as observed in certain split constructs (6). While this $K_{D}{ }^{\prime}$ is not straightforward to measure biochemically in vitro due to the overall irreversible nature of complementation, our steady state flow cytometry analysis provides a reliable way to characterize it in cells. Our model also shows that the overall complemented signal is determined not only thermodynamically by the initial binding affinity and local fragment concentrations, but also kinetically by the rates of folding, chromophore maturation and protein degradation. 
The assisted complementation demonstrated by SpyTag/SpyCatcher presents a simple way to improve complementation efficiency between FP fragments. This strategy can be expanded to multicolor imaging using $\mathrm{FP}_{11}$ tagging using orthogonal binder/tag pairs such as SnoopTag/SnoopCatcher (engineered by splitting an adhesin from Streptococcus pneumoniae (26)) or SsrA/SspB (a degradation tag and its adaptor protein from bacterial CIpX ATPase (27, 28)).

The substantial improvement of complementation efficiency in sfCherry $3_{1-10 / 11}$ is beneficial for a wide variety of applications ranging from protein labeling to scaffolding protein complexes, as well as monitoring cell-cell connections. A highly efficient complementation process not only guarantees an enhanced overall brightness, but also enables us to tune down the expression level of $\mathrm{FP}_{1-10}$ fragments which might otherwise exhaust the cellular machinery that maintaining the protein homeostasis (biogenesis, folding, trafficking and degradation of proteins). This benefit is essential in scenarios that are sensitive to the expression of exogenous proteins, such as tagging endogenous proteins in embryos. Moreover, our engineering platform based on pETDuet vector can be utilized to optimize other self-associating split FPs with insufficient complementation efficiency, such as mNeonGreen2 ${ }_{1-10 / 11}$.

Utilizing the split sfCherry31-10/11 construct, we have established a completely orthogonal, redcolored trans-synaptic marker NLG-1-CLASP in addition to the original green fluorescent transsynaptic marker NLG-1 GRASP. Similar cyan and yellow trans-synaptic markers (called dualeGRASP) have recently been generated in vertebrates (29). The use of NLG-1 CLASP with these cyan and yellow trans-synaptic markers has the potential to allow simultaneous and differential labeling of synapses between a single neuron and three synaptic partners in live animals. Since many synaptic connections within the nematode have overlapping localizations within the nerve ring and other nerve bundles $(11,12)$, this tool will allow us to accurately visualize multiple subsets of a single neuron's connections. These tools may similarly be of use in densely innervated regions of the vertebrate nervous system, such as the hippocampus and cortex. Thus, we propose that NLG-1 CLASP will be a powerful tool with which to probe the development and plasticity of neural circuits within live animals.

\section{Materials and Methods}

Molecular cloning. 
The DNA sequence of SpyCatcher 002 and SpyTag 002 (based on the reported sequence from (17)) were directly synthesized (Integrated DNA Technologies, IDT). The DNAs of histone H2B, TagBFP and mIFP were subcloned from mEmerald, TagBFP or mIFP fusion plasmids (cDNA source: the Michael Davidson Fluorescent Protein Collection at the UCSF Nikon Imaging Center) using Phusion High-Fidelity DNA Polymerase (Thermo Scientific). The P2A sequence are GCTACTAACTTCAGCCTGCTGAAGCAGGCTGGAGACGTGGAGGAGAACCCTGGACCT. The lentiviral plasmids pSFFV-GFP 1 -10, pSFFV-mNG2 ${ }_{1-10}$ and pSFFV-sfCherry2 ${ }_{1-10}$ were generated in our previous research (9). To build three pSFFV-mIFP-P2A-FP 11 -SpyCatcher constructs (used in Figs. 1A-C), three DNA fragments encoding mIFP, P2A-FP 11 and SpyCatcher were ligated into linearized pSFFV vector (BamHI/Notl) using In-Fusion HD Cloning kit (Clontech) within one reaction. To construct pSFFV-TagBFP-P2A-sfCherry2 1 -10 plasmid (used in Fig. 1D), two DNA fragments encoding TagBFP or P2A were ligated in to linearized pSFFV-sfCherry2 ${ }_{1-10}$ vector using In-Fusion. To generate pcDNA-SpyCatcher-6aa-sfCherry2 1 -10 and pcDNA-SpyCatcher-15aa-sfCherry2 ${ }_{1-10}$ plasmids (used in Figs. 2A-B), PCR amplicons encoding SpyCatcher or sfCherry2 ${ }_{1-10}$ were cloned into digested pcDNA3.1 vectors (HindIII/BamHI), and the different linkers were achieved through designing overlapping primers with various linker lengths. To make pSFFV-SpyTag-sfCherry $2{ }_{11}$-TagBFP and pSFFV-SpyTagsfCherry2 ${ }_{11}$-TagBFP-H2B (used in Figs. 2B-C), DNA fragments encoding SpyTag-sfCherry2 ${ }_{11}$, TagBFP and H2B were ligated in to linearized pSFFV vector (BamHI/Notl) through In-Fusion.

The pETDuet-1 vector was kindly donated by Dr. Alexander Kintzer from Dr. Robert Stroud's laboratory at UCSF. We generated the initial plasmid for mutagenesis screening by two rounds of In-Fusion ligation reaction: inserting the PCR amplicon encoding sfCherry 2 1-10 into the first multiple cloning site (MCS) of pETDuet-1 digested by Ncol, followed by inserting the DNA sequence encoding sfCherry ${ }_{11}$-SpyCatcher into the second MCS of pETDuet-1 digested by Ndel. The Ncol restriction site preserved in the final product, but the Ndel restriction site was destroyed. For the mammalian expression and lentiviral production, DNAs of sfCherry $3 \mathrm{C}_{1-10}$ were directly PCR amplified from identified pETDuet-1 construct (final mutant) and cloned into the lentiviral pSFFV vector (BamHI/Notl). To generate the sfCherry3 $\mathrm{V}_{1-10}$ variant, we introduced the point mutation L83W into pSFFV-sfCherry3 $\mathrm{C}_{1-10}$ plasmid using QuikChange II Site-Directed Mutagenesis Kit (Agilent Technologies). For the complete nucleotide sequence of sfCherry3 $\mathrm{C}_{1}$ 10, sfCherry3 $\bigvee_{1-10}$, SpyCatcher 002 and SpyTag 002, see Supplementary Table 1. 
Constructs used in the NLG-1 CLASP application were generated using standard molecular techniques. To generate ${ }_{p} g p a-6:: n / g-1:: s f C h e r r y 3 C_{1-10}$ construct (MVC227), sfCherry3 $C_{1-10}$ was amplified from $p S F F V$-sfCherry $3 C_{1-10}$ using the following primers: MVP846 (AGCTGCTAGCATGGAACGCATTTATCTTCTTCTCCTTCTTTTTCTGCCCAGGATACGATCC ATGGAGGAGGACAACATGG) and MVP847 (TCCGGAGCTCGTCCTCGTTGTGGCTGGT). The fragment was subcloned into $p g p a-6:: n / g-1:: G F P_{1-10}(\mathrm{MVC} 6)(20)$, replacing $\mathrm{GFP}_{1-10}$, using the Nhel and Sacl sites. To generate the ${ }_{p} f l p-18:: n / g-1::$ sfCherry2 ${ }_{11}$ (MVC228) construct, sfCherry $2_{11}$ was amplified from $\mathrm{H}_{2} B$-sfCherry $2_{11}$ using the following primers: MVP848 (AGCTGCTAGCATGGAACGCATTTATCTTCTTCTCCTTCTTTTTCTGCCCAGGATACGATCCT ACACCATCGTGGAGCAGT) and MVP849 (TCCGGAGCTCGGTGCTGTGTCTGGCCTC). The fragment was subcloned into $p$ flp-18::nlg-1::GFP 11 (MVC12)(20), replacing GFP 11 , using the Nhel and Sacl sites.

\section{Cell culture and lentiviral production.}

Human HEK 293T cells (UCSF cell culture facility) were maintained in Dulbecco's modified Eagle medium with high glucose (Gibco), supplemented with $10 \%$ (vol/vol) FBS and $100 \mu \mathrm{g} / \mathrm{ml}$ penicillin/streptomycin (UCSF Cell Culture Facility). U2OS cells (American Type Culture Collection, Manassas, VA) were cultured in DMEM media, supplemented with $10 \%$ fetal bovine serum. All cells were grown at $37{ }^{\circ} \mathrm{C}$ and $5 \% \mathrm{CO}_{2}$ in a humidified incubator. For the lentiviral production, $1 \times 10^{6}$ HEK 293 T cells were plated into T25 one day prior to transfection. $430 \mathrm{ng}$ of pMD2.G plasmid, $3600 \mathrm{ng}$ of pCMV-dR8.91 plasmid and $4100 \mathrm{ng}$ of the lentiviral plasmid

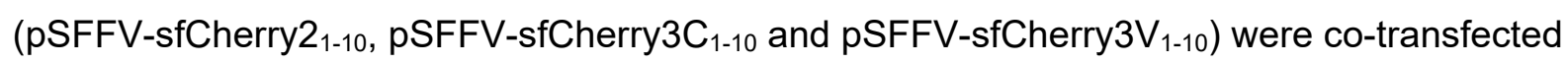
into HEK 293T cells using FuGENE HD (Promega) following the manufacturer's recommended protocol. The virus containing supernatant is harvested $48 \mathrm{~h}$ after transfection and were centrifuged to pellet any packaging cells. Virus containing medium is used immediately or stored in $-80^{\circ} \mathrm{C}$ freezer for future use.

For single-molecule brightness measurement sample preparation, U2OS cells were grown in 24-well plates with \#1.5 glass coverslip bottoms (In Vitro Scientific) and transfected $\sim 24$ hours before measurement using GenJet transfection reagent (SignaGen Laboratories) according to the manufacturer's instructions. Immediately before measurement, the growth media was exchanged with PBS buffer with calcium and magnesium (Gibco).

\section{Sample preparation and data analysis in flow cytometry.}


To characterize the relationship between complementation efficiency and expression level in split GFP, split mNG2 and split sfCherry2, we made pSFFV-mIFP_P2A_full-length-FP and pSFFV-mIFP_P2A_FP 11 -SpyCatcher constructs. Corresponding to each scatter plots in Figures 1B-D, $3 \times 10^{4}$ HEK 293T cells grown on 48-well plate (Eppendorf) were co-transfected with (B) left: 100 ng pSFFV-mIFP_P2A_GFP ${ }_{11}$-SpyCatcher with 200 ng pSFFV-GFP 1 10, right: 100 ng pSFFV-mIFP_P2A_GFP[full-length] with 200 ng pSFFV-GFP ${ }_{1-10}$; (C) left: 100 ng pSFFVmIFP_P2A_mNG2 ${ }_{11}$-SpyCatcher with 200 ng pSFFV-mNG2 ${ }_{1-10}$, right: 100ng pSFFVmIFP_P2A_mNG2[full-length] with 200 ng pSFFV-mNG2 $2_{1-10}$; (D) left: 100 ng pSFFVmIFP_P2A_sfCherry2 11 -SpyCatcher with 200 ng pSFFV-sfCherry2 ${ }_{1-10}$, right: 100 ng pSFFVmIFP_P2A_sfCherry2[full-length] with 200 ng pSFFV-sfCherry2 1 10. In Figure 1E, same cells were co-transfected with 100 ng pSFFV-mIFP_P2A_sfCherry2 ${ }_{11}-S p y C a t c h e r$ and $200 \mathrm{ng}$ pSFFV-sfCherry2 1 -10-TagBFP.

To test the Spy002 pair assisted complementation in sfCherry2, we built two tandem-binder constructs (pcDNA-SpyCatcher-6aa-sfCherry2 ${ }_{1-10}$ and pcDNA-SpyCatcher-15aa-sfCherry2 ${ }_{1-10}$ ) and two tandem-tag constructs (pSFFV-SpyTag-sfCherry $2{ }_{11}$-TagBFP and pSFFV-sfCherry $2{ }_{11}$ SpyTag-TagBFP). We then combinatorially co-transfected the same cells with 100ng tag construct plus $200 \mathrm{ng}$ binder construct. In Figure 2B, to compare the complementation efficiency with or without Spy pair interaction, cells were co-transfected with either 100 ng pSFFV-SpyTagsfCherry2 ${ }_{11}$-TagBFP plus 200 ng pcDNA-sfCherry2 1 -10, or 100 ng pSFFV-SpyTag-sfCherry2 ${ }_{11}$ TagBFP plus 200 ng SpyCatcher-6aa-sfCherry2 1 -10.

To validate the increased complementation of split sfCherry3 variants versus split sfCherry2, we made pSFFV-sfCherry3 $\mathrm{C}_{1-10}$ and pSFFV-sfCherry3 $\mathrm{V}_{1-10}$ constructs. Corresponding to each scatter plots in Figure 3E, same cells were co-transfected using with (left) 100ng pSFFVmIFP_P2A_sfCherry2 ${ }_{11}$-SpyCatcher with 200ng pSFFV-sfCherry2 ${ }_{1-10}$; (middle) 100ng pSFFVmIFP_P2A_sfCherry2 ${ }_{11}$-SpyCatcher with 200ng pSFFV-sfCherry3C $1-10$; (right) 100ng pSFFVmIFP_P2A_sfCherry2 ${ }_{11}$-SpyCatcher with 200ng pSFFV-sfCherry3V ${ }_{1-10}$.

For flow cytometry analysis, 48 hours after transfection, transfected HEK 293T cells were digested with Trypsin-EDTA (0.25\%) (Gibco) into single cells and re-suspended in $0.5 \mathrm{ml}$ PBS solution. Analytical flow cytometry was carried out on a LSR II instrument (BD Biosciences) and cell sorting on a FACSAria II (BD Biosciences) in Laboratory for Cell Analysis at UCSF. Flow cytometry data analysis (gating and plotting) was conducted using the FlowJo software (FlowJo LLC). 


\section{Single-molecule brightness measurement and data analysis.}

Fluorescence brightness measurements were carried out on a homebuilt two-photon microscope, which has been previously described (30). Pulsed laser light (100 fs pulses with a repetition frequency of $80 \mathrm{MHz}$ ) from a mode-locked Ti-Sapphire laser (Mai-Tai, Spectra Physics) was focused by a $63 \mathrm{X}$ C-Apochromat water immersion objective (NA = 1.2, Zeiss) to create two-photon excitation. The emitted fluorescence was collected by the same objective and separated from the excitation light by a dichroic mirror (675DCSXR, Chroma Technology). The fluorescence emission was separated into two detection channels by a 580nm dichroic mirror (585DCXR, Chroma Technology), and the green channel was further filtered by an 84nm-wide bandpass filter centered at $510 \mathrm{~nm}$ (FF01-510/84-25, Semrock). Avalanche photodiodes (SPCM-AQ-14, Perkin-Elmer) detected the fluorescence signal, and photon counts were recorded by a data acquisition card (FLEX02, Correlator.com) for $\sim 60$ seconds with a sampling frequency of $200 \mathrm{kHz}$. All measurements were carried out at an excitation wavelength of 1000 $\mathrm{nm}$ and a measured power after the objective of $\sim 0.46 \mathrm{~mW}$. The photon count record was analyzed to recover Mandel's $Q$ parameter as previously described (31) using programs written for IDL 8.7 (Research Systems, Inc.). The $Q$ value is converted into brightness $\lambda$, which represents the average fluorescence intensity per molecule, using the relation $Q=\gamma_{2} \lambda T$, where $T$ represents the sampling time and $\gamma_{2}$ is a shape-dependent factor whose value has been determined as described previously (32).

\section{Mutagenesis and screening.}

The amino-acid sequence of sfCherry2 and the split site were from our previous published literature (9). The sfCherry2 ${ }_{1-10}$ sequence was subjected to random mutagenic PCR (forward primer: AGGAGATATACCATGGAGGAGGACAAC, reverse primer:

CTGCTGCCCATGTCAGTCCTCGTTGTG) using the GeneMorph II Random Mutagenesis Kit (Agilent Technologies). A high mutation rate protocol suggested in the instruction manual was adapted, with an initial target DNA amount of $0.2 \mu \mathrm{g}$ and 30 -cycle amplification. The cDNA library pool was gel-purified and ligated into a PCR-linearized $p E T D u e t$ vector (forward primer: TGACATGGGCAGCAGCCA , reverse primer:

CATGGTATATCTCCTTCTTAAAGTTAAACAAAATTATTTCTAGAGG. The product only contains the sfCherry2 ${ }_{11}$-SpyCatcher coding sequence in the $2^{\text {nd }}$ MCS but not the sfCherry ${ }_{1-10}$ in the $1^{\text {st }}$ MCS.) using In-Fusion. The plasmid pool was then transformed into E.coli BL21 (DE3) electrocompetent cells (Lucigen) by electroporation using the Gene Pulser Xcell ${ }^{\mathrm{TM}}$ 
Electroporation Systems (BioRad). The expression library was evenly plated on nitrocellulose membrane (Whatman, $0.45 \mu \mathrm{m}$ pore size), which was sitting on an LB-agar plate with $30 \mathrm{mg} / \mathrm{ml}$ kanamycin. After overnight growth at $37^{\circ} \mathrm{C}$, the nitrocellulose membrane was carefully transferred onto a new LB-agar plate containing $1 \mathrm{mM}$ Isopropyl- $\beta$-D-thiogalactoside (IPTG) and $30 \mathrm{mg} / \mathrm{ml}$ kanamycin and cultured for another 3-6 hours at $37^{\circ} \mathrm{C}$ to induce the protein production. We performed the clone screening by imaging the IPTG-containing LB-agar plate using a BioSpectrum Imaging System (UVP). The brightest candidates in each library were pooled (typically $\sim 20$ from approximately 10,000 colonies) and served as templates for the next round of directed evolution. The DNA sequences of selected constructs were confirmed by sequencing (Quintara Biosciences). For DNA shuffling, we adopted the protocol described in $\mathrm{Yu}$ et al (33). Specifically, we PCR amplified the brightest six sfCherry31-10 variants (forward primer: AGGAGATATACCATGGAGGAGGACAAC, reverse primer: CTGCTGCCCATGTCAGTCCTCGTTGTG) from the last round of random mutagenesis screening. PCR products of $651 \mathrm{bp}$ were purified from $1 \%$ agarose gels using zymoclean gel DNA gel recovery kit (Zymo Research). The DNA concentrations were measured in Nanodrop and the fragments were mixed at equal amounts for a total of $\sim 2 \mu \mathrm{g}$. The mixture was then digested with 0.5 unit DNase I (New England Biolabs) for 13min and terminated by heating at $95^{\circ} \mathrm{C}$ for $10 \mathrm{~min}$. The DNase I digests were run on a $2 \%$ agarose gel, and the band with a size of 50-100 bp was selected and purified. $10 \mu \mathrm{l}$ of purified fragments was added to $10 \mu \mathrm{l}$ of Phusion High-Fidelity PCR Master Mix and reassembled with a PCR program of 30 cycles, with each cycle consisting of $95^{\circ} \mathrm{C}$ for $60 \mathrm{~s}, 50{ }^{\circ} \mathrm{C}$ for $60 \mathrm{~s}$ and $72{ }^{\circ} \mathrm{C}$ for $30 \mathrm{~s}$. After reassembly, $1 \mu \mathrm{l}$ of this reaction was amplified by PCR. The shuffled library was then transformed, expressed and screened as described above. After the directed evolution was saturated (no apparent fluorescence increase in the induced colonies), the brightest clone was selected and the DNA sequences of the constructs were confirmed by sequencing (Quintara Biosciences). The emission spectra of split sfCherry variants (in E.coli solution culture expressing FPs) were measurement on a Synergy ${ }^{\mathrm{TM}} \mathrm{H} 4$ Hybrid Multi-Mode Microplate Reader from UCSF Center for Advanced Technology.

\section{sgRNA production, RNP nucleofection and lentiviral transduction}

We purchased synthetic single strand DNA oligos from Integrated DNA Technologies (IDT). We prepared sgRNAs and Cas9/sgRNA RNP complexes following our published methods (4). Specifically, sgRNAs were obtained by in vitro transcribing DNA templates containing a T7 promoter (TAATACGACTCACTATAG), an sgRNA scaffold region and the gene-specific $20 \mathrm{nt}$ 
sgRNA sequence. DNA templates were produced by overlapping PCR using a set of 4 primers: 3 common primers (forward primer T25: 5'- TAA TAC GAC TCA CTA TAG -3'; reverse primer BS7: 5'- AAA AAA AGC ACC GAC TCG GTG C -3' and reverse primer ML611: 5'- AAA AAA AGC ACC GAC TCG GTG CCA CTT TTT CAA GTT GAT AAC GGA CTA GCC TTA TTT AAA CTT GCT ATG CTG TTT CCA GCA TAG CTC TTA AAC -3') and one gene-specific primer (forward primer 5'- TAA TAC GAC TCA CTA TAG NNN NNN NNN NNN NNN NNN NNG TTT AAG AGC TAT GCT GGA A -3'). For each template, a $50 \mu \mathrm{L}$ PCR was performed with Phusion $®$ High-Fidelity PCR Master Mix (New England Biolabs) reagents with the addition of 1 $\mu \mathrm{M}$ T25, $1 \mu \mathrm{M}$ BS7, 20 nM ML611 and 20 nM gene-specific primer. The PCR product was purified and eluted in $12 \mu \mathrm{L}$ of RNAse-free DNA buffer. Next, a 100- $\mu \mathrm{L}$ in vitro transcription reaction was performed with $\sim 300 \mathrm{ng}$ DNA template from PCR product and $1000 \mathrm{U}$ of T7 RNA polymerase in buffer containing $40 \mathrm{mM}$ Tris $\mathrm{pH}$ 7.9, $20 \mathrm{mM} \mathrm{MgCl}_{2}, 5 \mathrm{mM}$ DTT, $2 \mathrm{mM}$ spermidine and $2 \mathrm{mM}$ of each NTP (New England BioLabs). Following a $4 \mathrm{~h}$ incubation at $37^{\circ} \mathrm{C}$, the sgRNA product was purified and eluted in $15 \mu \mathrm{L}$ of RNAse-free RNA buffer. The sgRNA was qualitychecked by running $5 \mathrm{pg}$ of the product on Mini-PROTEAN TBE Precast Gels (Bio-Rad Laboratories) at 200-voltage for 60 80 $\mathrm{min}$.

For the knock-in of sfCherry $2{ }_{11}$ into HEK 293 T wild-type (WT) cells, 200-nt homology-directed recombination (HDR) templates were ordered in single-stranded DNA (ssDNA) from IDT. For the complete set of DNA sequence used for sgRNA in vitro transcription or HDR templates, see Supplementary Tables 2 and 3. Cas9 protein (pMJ915 construct, containing two nuclear localization sequences) was expressed in E.coli and purified by the University of California Berkeley Macrolab. HEK 293T WT cells were treated with $200 \mathrm{ng} / \mathrm{mL}$ nocodazole (Sigma) for $\sim 17$ hours before electroporation to increase HDR efficiency. 100 pmol Cas 9 protein and 130 pmol sgRNA were assembled into Cas9/sgRNA RNP complexes just before nucleofection and combined with $150 \mathrm{pmol}$ HDR template in a final volume of $10 \mu \mathrm{L}$. Electroporation was performed in an Amaxa 96-well shuttle Nuleofector device (Lonza) using SF-cell line reagents (Lonza). Nocodazole-treated cells were resuspended to $10^{4}$ cells/ $\mu \mathrm{L}$ in SF solution immediately prior to electroporation. For each sample, $20 \mu \mathrm{L}$ of cells was added to the $10 \mu \mathrm{L} \mathrm{RNP/template}$ mixture. Cells were immediately electroporated using the CM130 program and transferred to 48well plate with pre-warmed medium. Electroporated cells were cultured and expanded for 7-10 days prior to lentiviral transduction.

One day before lentiviral transduction, knocked-in cells well split and seeded in 12-well plate at $6 \sim 8 \times 10^{4}$ per well for 4 wells. The confluency should reach $70 \sim 80 \%$ on the day of transduction. 
The lentivirus titer of sfCherry $2_{1-10}$, sfCherry $3 C_{1-10}$ or sfCherry3 $V_{1-10}$ was quantified independently by Lenti-X GoStix Plus kit (Takara, Cat\# 631280) immediately before infection. And the supernatant was diluted (around 1:5 dilution) with fresh medium to make sure the final virus concentrations are the same across groups. The experimental groups were treated with $1 \mathrm{ml}$ diluted sfCherry2 ${ }_{1-10}$, sfCherry $3 \mathrm{C}_{1-10}$ or sfCherry3 $\mathrm{V}_{1-10}$ viral supernatant supplemented with 10 ug polybrene (MilliporeSigma) respectively. The negative control was treated with fresh medium supplemented with the same concentration of polybrene. 24-hour after infection, the viral supernatant was swapped with fresh medium. After another 48 to 72 hours, the infected cells were harvested for flow cytometry analysis and cell sorting.

\section{Fluorescence Microscopy}

To validate the Spy-assisted complementation can improve the overall brightness in protein labeling (Fig. 2C), we transfected HEK 293T cells (1.5 X 104 per well) grown on an 8-well glass bottom chamber (Thermo Fisher Scientific) using FuGene HD (Promega). In order to achieve better cell attachment, 8-well chamber was coated with Fibronectin (Sigma-Aldrich) for 45 min before seeding cells. Total plasmid amount of $180 \mathrm{ng}$ per well with the $\mathrm{FP}_{11}$ to $\mathrm{FP}_{1-10}$ ratio in 1:2 was used to achieve optimal labeling. 48 hours after transfection, cells were fixed with $4 \%$ paraformaldehyde and then imaged on a Nikon Ti-E inverted wide-field fluorescence microscope equipped with an LED light source (Excelitas X-Cite XLED1), a 40×0.55 NA air objective (Nikon), a motorized stage (ASI) and an sCMOS camera (Tucsen).

Live-cell imaging (Figs. 4B-G) of sorted successful knock-in cells (with either sfCherry3 $\mathrm{C}_{1-10}$ or sfCherry3 $\mathrm{V}_{1-10}$ infection) was acquired on an inverted Nikon Ti-E microscope (UCSF Nikon Imaging Center), a Yokogawa CSU-W1 confocal scanner unit, a Plan Apo VC 100× 1.4 NA oil immersion objective, a stage incubator, an Andor Zyla 4.2 sCMOS camera and MicroManager2.0 software. Fluorescence recovery after photobleaching (FRAP) experiment (fig. S1) was performed on the same microscopy with a Vortran $473 \mathrm{~nm}$ laser for photobleaching and a Rapp Optoelectronic UGA-40 photobleaching system (COM5). The frame interval of the movie is $2.5 \mathrm{~s}$ and the total length is $10 \mathrm{~min}$. The photobleaching was conducted by scanning the $473 \mathrm{~nm}(50 \mathrm{~mW})$ laser over the region of interest at the $20 \mathrm{~s}$ of the movie. For optimal cell attachment, 8-well glass bottom chamber was coated with Fibronectin (Sigma-Aldrich) for 45 min before seeding sorted endogenously tagged HEK 293T cells. A background image (taken at the same imaging condition without putting on any sample) was subtracted from the live-cell 
microscopy images using the ImageJ software. Analysis of fluorescence microscopy images were performed in ImageJ.

\section{Generation of NLG-1 CLASP in C. elegans}

All C. elegans strains were maintained using standard protocols(34) and were raised on $60 \mathrm{~mm}$ Nematode Growth Media plates seeded with OP50 Escherichia coli at $20^{\circ} \mathrm{C}$. Wild-type strains were C. elegans variety Bristol, strain N2, and the mutant strain used for this study was clr1(e1745) II. Transgenic strains include wyEx1982 (20), wyEx1982; clr-1, iyEx368 and iyEx368; clr-1. wyEx1982 contains extra-chromosomal PHB-AVA NLG-1 GRASP marker (pgpa-6::nlg-

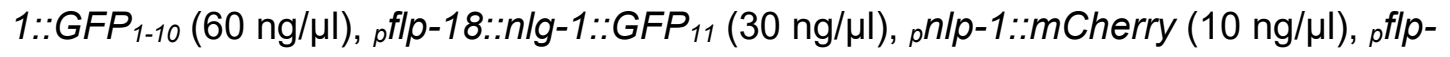
18::mCherry $(5 \mathrm{ng} / \mu \mathrm{l})$ and $\left.{ }_{p} \mathrm{Odr}-1:: D s R e d I I(20 \mathrm{ng} / \mu \mathrm{l})\right)$. iyEx368 contains the extra-chromosomal PHB-AVA NLG-1 CLASP marker ( $g$ ga-6::nlg-1::sfCherry3C $1-10(68 \mathrm{ng} / \mu \mathrm{l})$, plp-18::nlg-

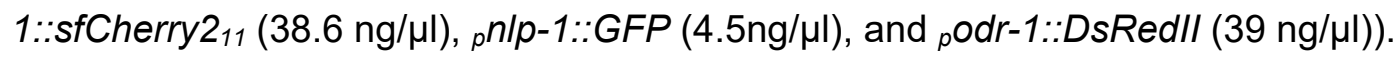

A Zeiss Axio Imager.A1 compound fluorescent microscope was used to capture images of live C. elegans under $63 \mathrm{X}$ magnification. Worms were paralyzed on $2 \%$ agarose pads using a 2:1 ratio of $0.3 \mathrm{M}$ 2,3-butanedione monoxime (BDM) and $10 \mathrm{mM}$ levamisole in M9 buffer. All micrographs taken of PHB-AVA NLG-1 GRASP and NLG-1 CLASP markers were of larval stage 4 animals. All data from micrographs were quantified using $\mathrm{NIH}$ ImageJ software. Intensity of PHB-AVA NLG-1 GRASP and PHB-AVA NLG-1 CLASP was measured as previously described $(13,35)$. Briefly, the intensity at each pixel within each synaptic puncta was measured using NIH ImageJ. To account for differences in background fluorescence, background intensity was estimated by calculating the minimum intensity value in a region immediately adjacent to the puncta. This minimum intensity value was then subtracted from the intensity for each pixel, and the sum of the adjusted values was calculated. For control, pictures of wild-type animals were also taken on the same day using the same settings.

\section{Acknowledgement}

We thank Dr. David Alexander Brown, Dr. Yina Wang for extensive discussion on data analysis, Dr. Bin Yang for help in fluorescence light microscopy, Alejandro Ramirez for preparing reagents and supplies, Dr. Kari Herrington for help in FRAP experiment, Dr. Noelle L'Etoile for advice on NLG-1 CLASP experiments, Dr. Xiaokun Shu for sharing the BioSpectrum Imaging System, and Dr. Joseph DeRisi for generously sharing the nucleofector device. This work is supported by the National Institutes of Health R21EB022798 and R01GM124334 (to B.H.), 
UCSF Program for Breakthrough Biomedical Research (Byers Award in Basic Science to B.H.), the National Institutes of Health R01GM064589 (to J.K. and J.D.M.), the National Institutes of Health R01NS087544 and SC3GM089595 (to M.V.), T34GM008253 MARC fellowship (to N.A.), and the National Science Foundation 1355202 (to M.V.). B.H. is a Chan Zuckerberg Biohub investigator.

\section{Competing Financial Interest}

The authors declare no competing financial interests.

\section{Author Contributions}

S.F. and B.H. conceived and designed the research; S.F. performed the flow cytometry, random mutagenesis and screening, CRISPR knock-in, FACS, confocal microscopy and FRAP experiments; J.K and J.D.M. performed single-molecule brightness measurement and analysis; S.F. and C.M. performed the molecular cloning and site-directed mutagenesis experiments; A.V., D.C.V., F.F., N.A. and M. V. perfored the NLG-1 CLASP experiments and analysis; S.F. and B.H. analyzed the data; S.F. and B.H. wrote the manuscript. 


\section{Figures}
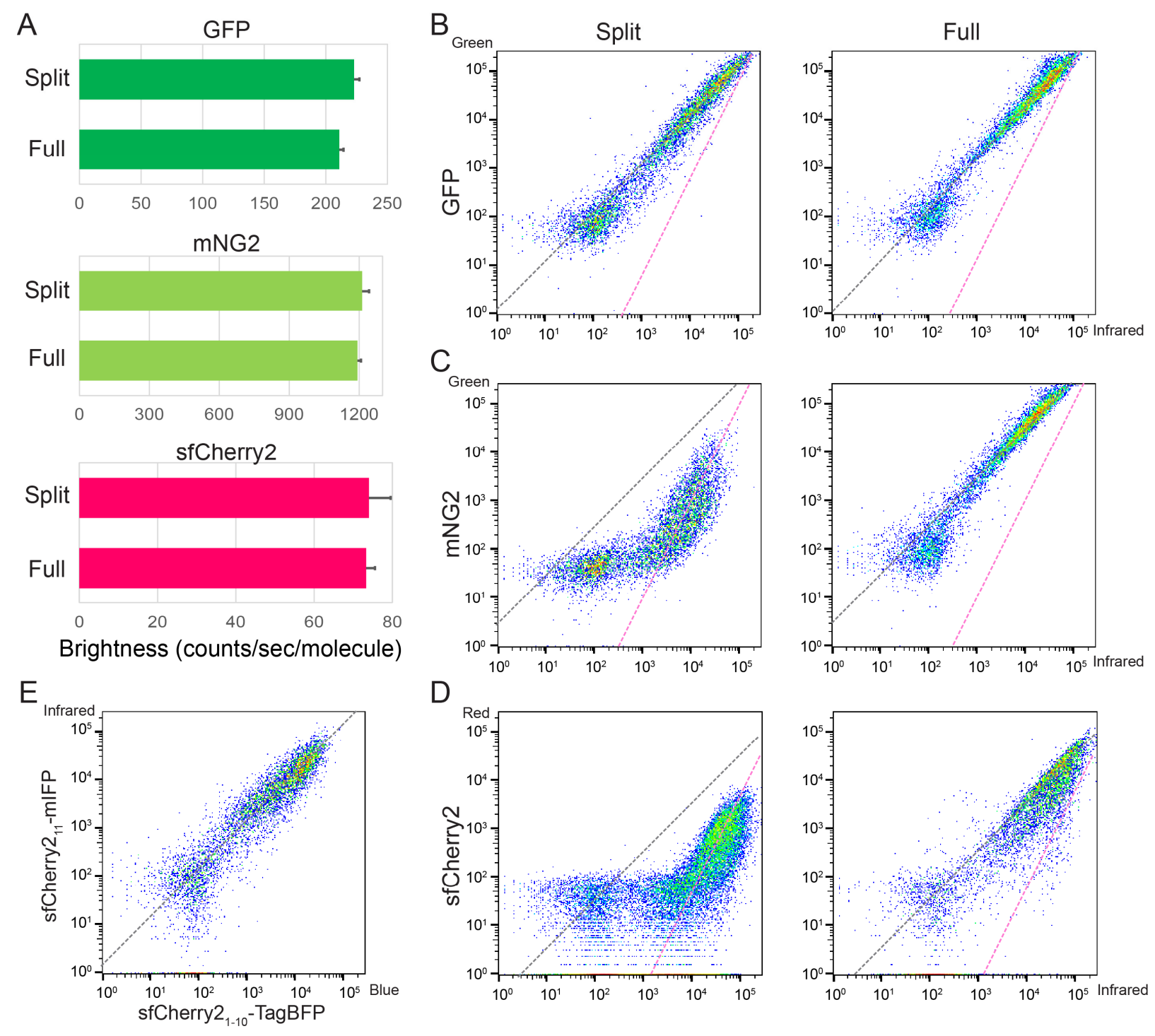

Fig. 1. Characterization of split fluorescent proteins. (A) Single molecule brightness measurement of split FPs and their full-length counterparts using fluorescence fluctuation spectroscopy. $N=10$. Error bars are standard deviations. (B to $\mathbf{D}$ ) Flow cytometry analysis of whole cell fluorescence in HEK 293T expressing either (B) GFP 1 -10/11, (C) mNG21-10/11, and (D) sfCherry $2_{1-10 / 11}$ or their full-length counterparts. The $x$-axis is the log-scale infrared fluorescence intensity indicating the expression level, and the $y$-axis is the log-scale green (or red) fluorescence intensity. The grey dashed trend lines have a slope of 1 and intercepts are set to best follow the points in the right (full-length) panels. The pink dashed trend lines have a slope of 2 and intercepts set to best follow the points in the left (split) panels with the exception of

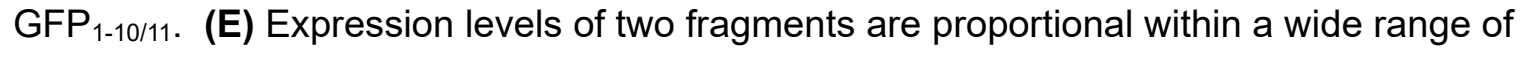
expression levels in a co-transfect experiment. The grey dashed trend line has a slope of 1. 
A
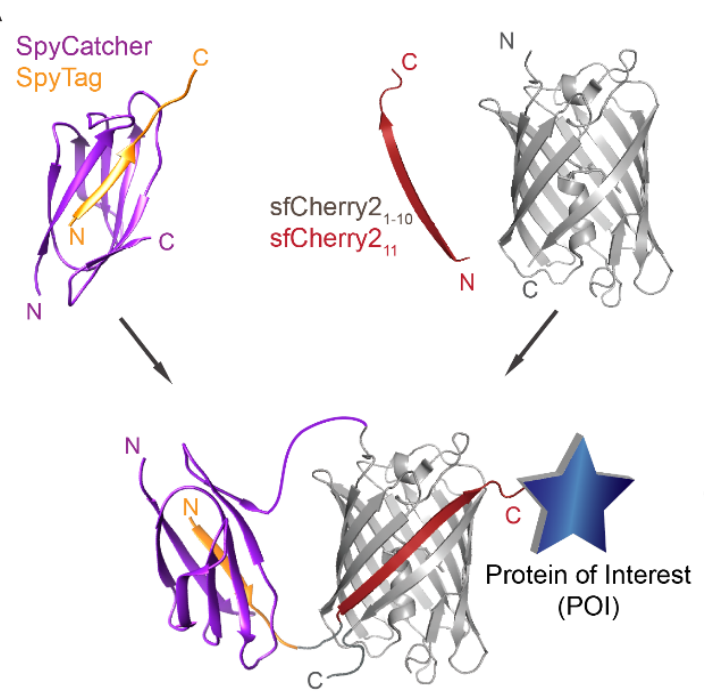

Binder:

SpyCatcher-6aa-sfCherry $2_{1-10}$

SpyCatcher-15aa-sfCherry2

SpyCatcher-15aa-sfCherry $2_{1-10}$
Tag:

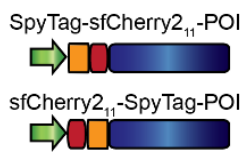

B
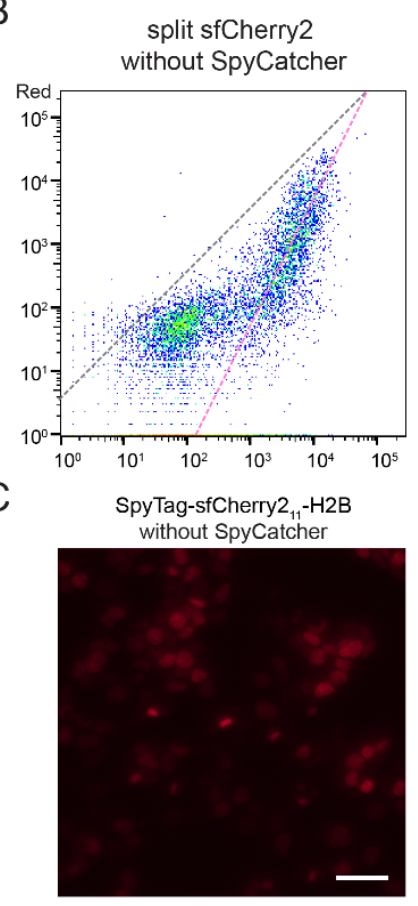

split sfCherry2

with SpyCatcher

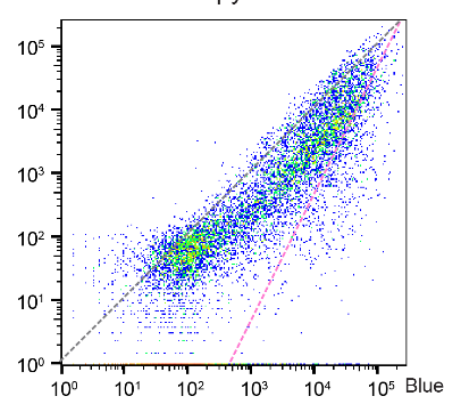

SpyTag-sfCherry ${ }_{11}-\mathrm{H} 2 \mathrm{~B}$ with SpyCatcher

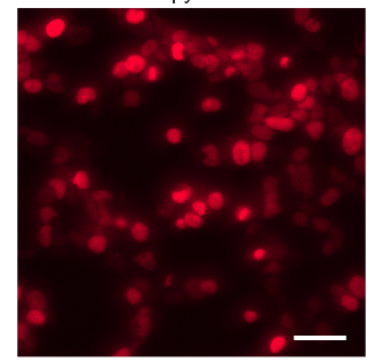

Fig. 2. SpyTag/SpyCatcher assisted complementation of split sfCherry21-10/11. (A)

Schematic diagram of SpyTag/SpyCatcher-assisted complementation and construct design. (B)

Flow cytometry analysis of whole cell fluorescence in HEK 293T cells expressing SpyTagsfCherry $2_{11}$-TagBFP with either sfCherry $2_{1-10}$ alone or with SpyCatcher-6aa-sfCherry2 ${ }_{1-10}$. The grey dashed trend line has a slope of 1 and the pink one has a slope of 2. (C) Fluorescence microscopy of sfCherry2 ${ }_{11}$ labeled H2B without (left) or with (right) the assistance of SpyTag/SpyCatcher interaction. The imaging condition and brightness/contrast range were set the same for better comparison. Scale bars: $50 \mu \mathrm{m}$. 


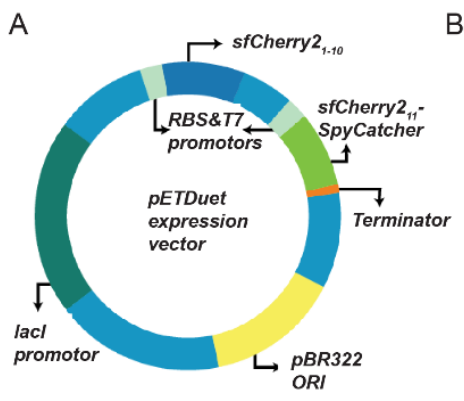

D Emission spectrum of sfCherry variants

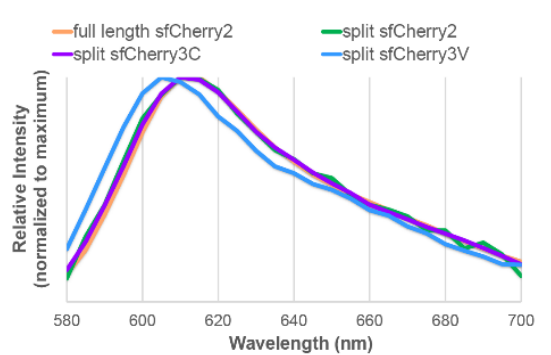

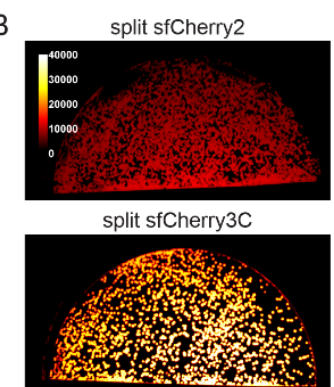

E

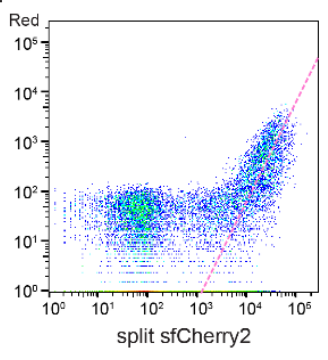

C
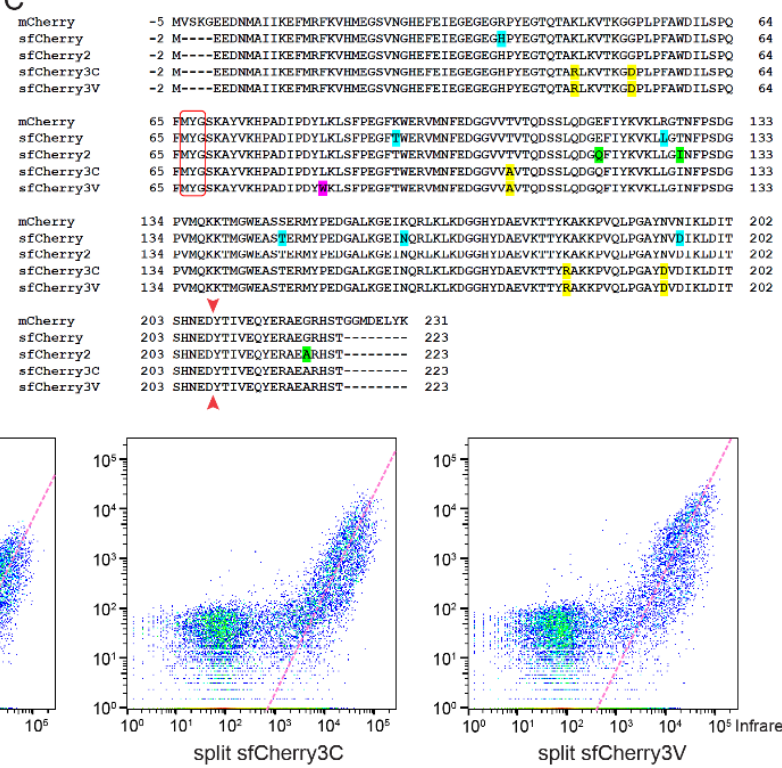

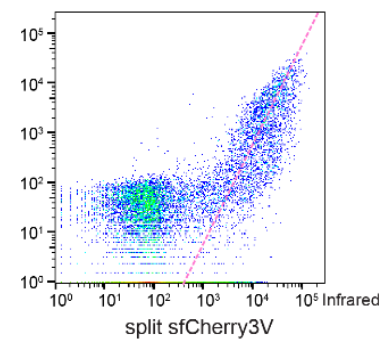

Fig. 3. Engineering and characterization of split sfCherry3. (A) Schematics of pETDuetbased engineering platform. (B) Fluorescence images of E.coli colonies expressing split sfCherry2 or sfCherry3C from the pETDuet constructs. (C) Protein sequence alignment of mCherry, sfCherry, sfCherry2, sfCherry3C and sfCherry3V. The amino acids forming the chromophore are indicated by a red box. The split site is indicated by the red arrow. Starting from mCherry, mutations introduced in sfCherry, sfCherry2, sfChery3C and sfCherry3V are highlighted in cyan, green, yellow and magenta, respectively. The overall alignment numbering follows that of sfCherry. (D) Emission spectra of sfCherry variants. (E) Flow cytometry analysis of whole cell fluorescence in HEK 293T cells expressing mIFP-P2A- sfCherry2 ${ }_{11}$-SpyCatcher and sfCherry $2_{1-10}$ (left)/ sfCherry3 $\mathrm{C}_{1-10}$ (middle)/ sfCherry3 $\mathrm{V}_{1-10}$ (right). The pink dashed lines are results from linear least-square fitting with a fixed slope of 2 (see fig. S4). 

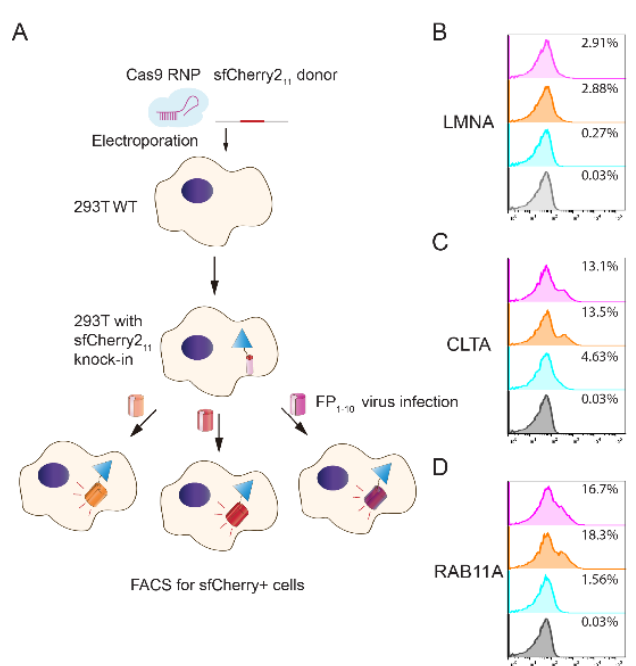
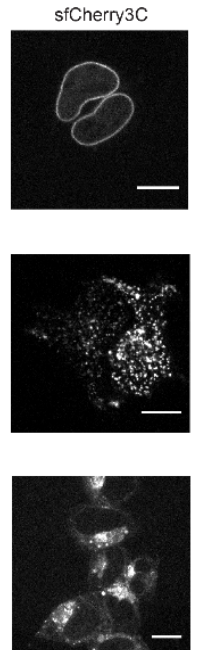
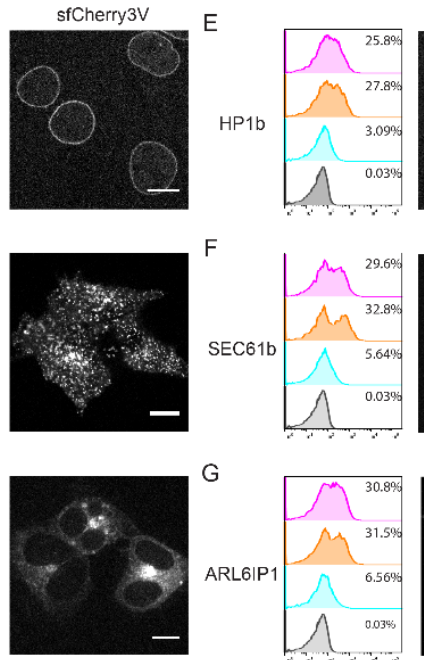
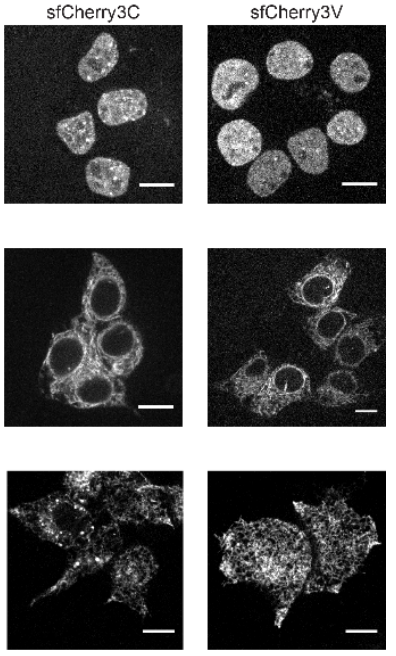

Fig. 4. Endogenous protein labeling in HEK 293T cells using sfCherry3 variants. (A) Schematic diagram of knock-in followed by virus infection and FACS enrichment. (B to $\mathbf{G})$ Analysis of FACS sorting efficiency in six targets, (B) lamin A/C, (C) clathrin light chain $A$, (D) RAB11A, (E) heterochromatin protein $1 \beta$, (F) ER translocon complex SEC61b and (G) ER tubule protein ARL6IP1, and visualization of sorted knock-in cells through confocal fluorescence microscopy. Scale bar: $10 \mu \mathrm{m}$. 
A

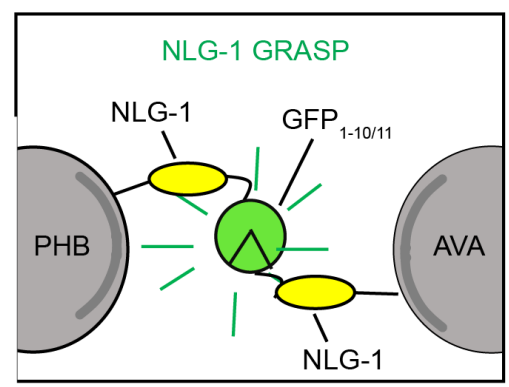

C

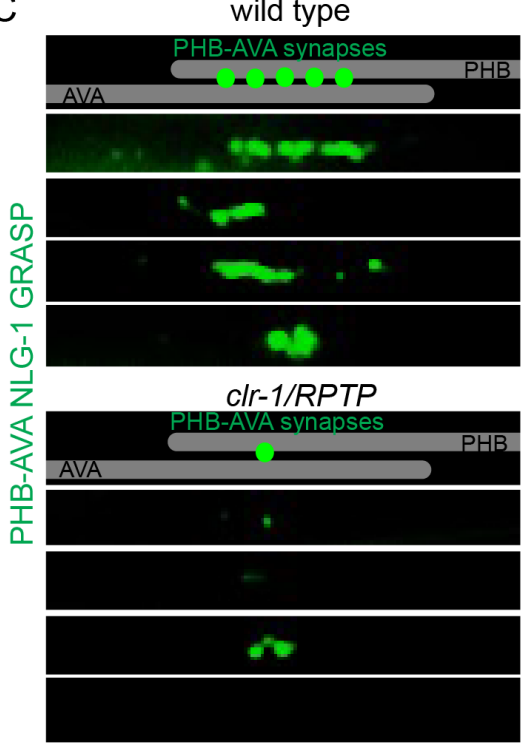

E

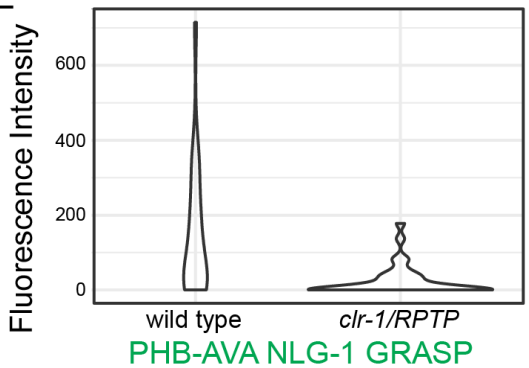

B

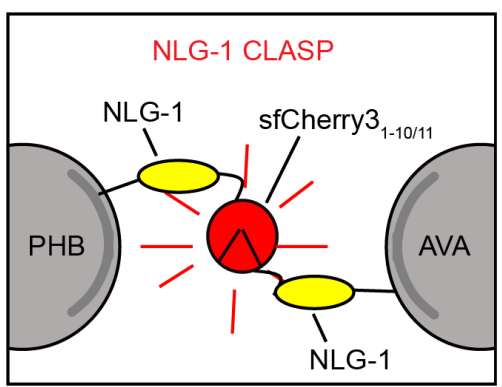

D

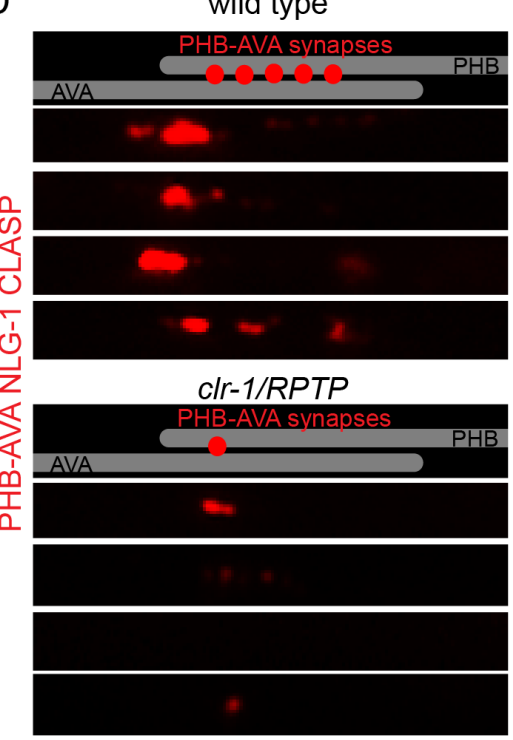

$\mathrm{F}$

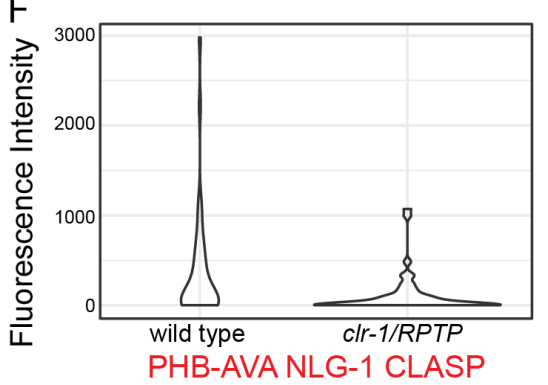

Fig. 5. NLG-1 CLASP visualizes specific subsets of synapses in live $C$. elegans. (A \& B)

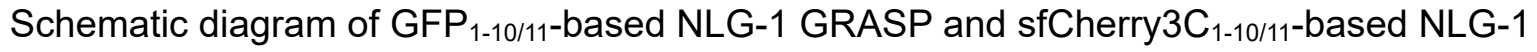
CLASP in PHB and AVA neurites. (C \& D) Schematics and micrographs of NLG-1 GRASP and NLG-1 CLASP specifically labelling synaptic contacts between PHB and AVA neurons in C. elegans. PHB-AVA NLG-1 GRASP and NLG-1 CLASP fluorescence intensity are dramatically reduced in the synaptic partner recognition mutant clr-1/RPTP. (E \& F) Quantification of the reduction in relative fluorescence intensities of NLG-1 GRASP and NLG-1 CLASP in clr-1/RPTP mutants. $N \geq 40$. 


\section{References}

1. S. Cabantous, T. C. Terwilliger, G. S. Waldo, Protein tagging and detection with engineered self-assembling fragments of green fluorescent protein. Nat Biotechnol 23, 102-107 (2005).

2. S. B. Van Engelenburg, A. E. Palmer, Imaging type-III secretion reveals dynamics and spatial segregation of Salmonella effectors. Nat Methods 7, 325-330 (2010).

3. D. Kamiyama et al., Versatile protein tagging in cells with split fluorescent protein. Nat Commun 7, 11046 (2016).

4. M. D. Leonetti, S. Sekine, D. Kamiyama, J. S. Weissman, B. Huang, A scalable strategy for high-throughput GFP tagging of endogenous human proteins. Proc Natl Acad Sci U S A 113, E3501-3508 (2016).

5. S. Cabantous et al., Recent advances in GFP folding reporter and split-GFP solubility reporter technologies. Application to improving the folding and solubility of recalcitrant proteins from Mycobacterium tuberculosis. J Struct Funct Genomics 6, 113-119 (2005).

6. W. Chun, G. S. Waldo, G. V. Johnson, Split GFP complementation assay: a novel approach to quantitatively measure aggregation of tau in situ: effects of GSK3beta activation and caspase 3 cleavage. J Neurochem 103, 2529-2539 (2007).

7. H. Ishikawa, F. Meng, N. Kondo, A. Iwamoto, Z. Matsuda, Generation of a dual-functional split-reporter protein for monitoring membrane fusion using self-associating split GFP. Protein Eng Des Sel 25, 813-820 (2012).

8. E. H. Feinberg et al., GFP Reconstitution Across Synaptic Partners (GRASP) defines cell contacts and synapses in living nervous systems. Neuron 57, 353-363 (2008).

9. S. Feng et al., Improved split fluorescent proteins for endogenous protein labeling. Nat Commun 8, 370 (2017).

10. D. Batan et al., A Multicolor Split-Fluorescent Protein Approach to Visualize Listeria Protein Secretion in Infection. Biophys J 115, 251-262 (2018).

11. J. G. White, E. Southgate, J. N. Thomson, S. Brenner, The structure of the nervous system of the nematode Caenorhabditis elegans. Philos Trans R Soc Lond B Biol Sci 314, 1-340 (1986). 
12. D. H. Hall, R. L. Russell, The posterior nervous system of the nematode Caenorhabditis elegans: serial reconstruction of identified neurons and complete pattern of synaptic interactions. J Neurosci 11, 1-22 (1991).

13. A. Varshney et al., The receptor protein tyrosine phosphatase CLR-1 is required for synaptic partner recognition. PLoS Genet 14, e1007312 (2018).

14. B. D. Slaughter, R. Li, Toward quantitative "in vivo biochemistry" with fluorescence fluctuation spectroscopy. Mol Biol Cell 21, 4306-4311 (2010).

15. B. Zakeri et al., Peptide tag forming a rapid covalent bond to a protein, through engineering a bacterial adhesin. Proc Natl Acad Sci U S A 109, E690-697 (2012).

16. V. Pessino, Y. R. Citron, S. Feng, B. Huang, Covalent Protein Labeling by SpyTagSpyCatcher in Fixed Cells for Super-Resolution Microscopy. Chembiochem 18, 1492-1495 (2017).

17. A. H. Keeble et al., Evolving Accelerated Amidation by SpyTag/SpyCatcher to Analyze Membrane Dynamics. Angew Chem Int Ed Engl 56, 16521-16525 (2017).

18. H. J. Carlson, D. W. Cotton, R. E. Campbell, Circularly permuted monomeric red fluorescent proteins with new termini in the beta-sheet. Protein Sci 19, 1490-1499 (2010).

19. H. B. Nguyen, L. W. Hung, T. O. Yeates, T. C. Terwilliger, G. S. Waldo, Split green fluorescent protein as a modular binding partner for protein crystallization. Acta Crystallogr $D$ Biol Crystallogr 69, 2513-2523 (2013).

20. J. Park et al., A conserved juxtacrine signal regulates synaptic partner recognition in Caenorhabditis elegans. Neural Dev 6, 28 (2011).

21. H. Lee, W. C. Oh, J. Seong, J. Kim, Advanced Fluorescence Protein-Based SynapseDetectors. Front Synaptic Neurosci 8, 16 (2016).

22. G. Jansen et al., The complete family of genes encoding $\mathrm{G}$ proteins of Caenorhabditis elegans. Nat Genet 21, 414-419 (1999).

23. C. Rogers et al., Inhibition of Caenorhabditis elegans social feeding by FMRFamide-related peptide activation of NPR-1. Nat Neurosci 6, 1178-1185 (2003).

24. T. K. Kerppola, Bimolecular fluorescence complementation: visualization of molecular interactions in living cells. Methods Cell Biol 85, 431-470 (2008). 
25. C. D. Hu, Y. Chinenov, T. K. Kerppola, Visualization of interactions among bZIP and Rel family proteins in living cells using bimolecular fluorescence complementation. $\mathrm{Mol}$ Cell $\mathbf{9}$, 789-798 (2002).

26. G. Veggiani et al., Programmable polyproteams built using twin peptide superglues. Proc Natl Acad Sci U S A 113, 1202-1207 (2016).

27. I. Levchenko, R. A. Grant, D. A. Wah, R. T. Sauer, T. A. Baker, Structure of a delivery protein for an AAA+ protease in complex with a peptide degradation tag. Mol Cell 12, 365372 (2003).

28. G. Guntas et al., Engineering an improved light-induced dimer (iLID) for controlling the localization and activity of signaling proteins. Proc Natl Acad Sci U S A 112, 112-117 (2015).

29. J. H. Choi et al., Interregional synaptic maps among engram cells underlie memory formation. Science 360, 430-435 (2018).

30. B. Wu, Y. Chen, J. D. Muller, Fluorescence fluctuation spectroscopy of mCherry in living cells. Biophys J 96, 2391-2404 (2009).

31. A. Sanchez-Andres, Y. Chen, J. D. Muller, Molecular brightness determined from a generalized form of Mandel's Q-parameter. Biophys J 89, 3531-3547 (2005).

32. P. J. Macdonald, Y. Chen, X. Wang, Y. Chen, J. D. Mueller, Brightness analysis by Z-scan fluorescence fluctuation spectroscopy for the study of protein interactions within living cells. Biophys J 99, 979-988 (2010).

33. D. Yu et al., An improved monomeric infrared fluorescent protein for neuronal and tumour brain imaging. Nat Commun 5, 3626 (2014).

34. S. Brenner, The genetics of Caenorhabditis elegans. Genetics 77, 71-94 (1974).

35. J. Park et al., A conserved juxtacrine signal regulates synaptic partner recognition in Caenorhabditis elegans. Neural Dev 6, 28 (2011). 


\section{Supplementary Figures}
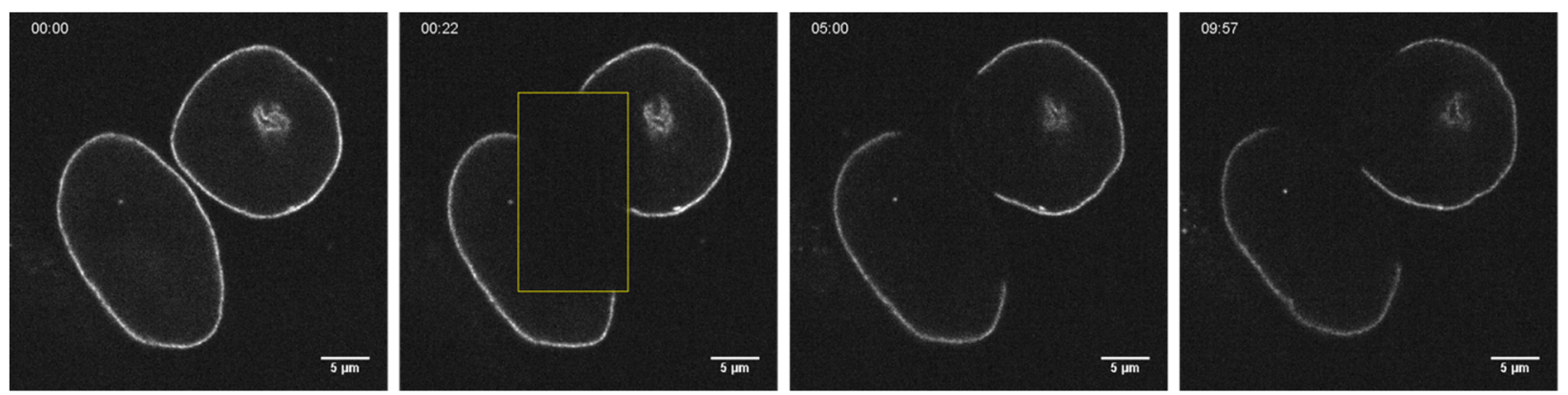

Supplementary Figure 1. Snapshots of fluorescence recovery after photobleaching (FRAP) experiment on endogenously mNeonGreen $2_{1-10 / 11}$ labeled Lamin A/C (nuclear envelope) in HEK 293T cells at different time points (before bleaching, $1^{\text {st }}$ frame, $5 \mathrm{~min}$ and $10 \mathrm{~min}$ after bleaching). The yellow square marks the photobleaching area. 

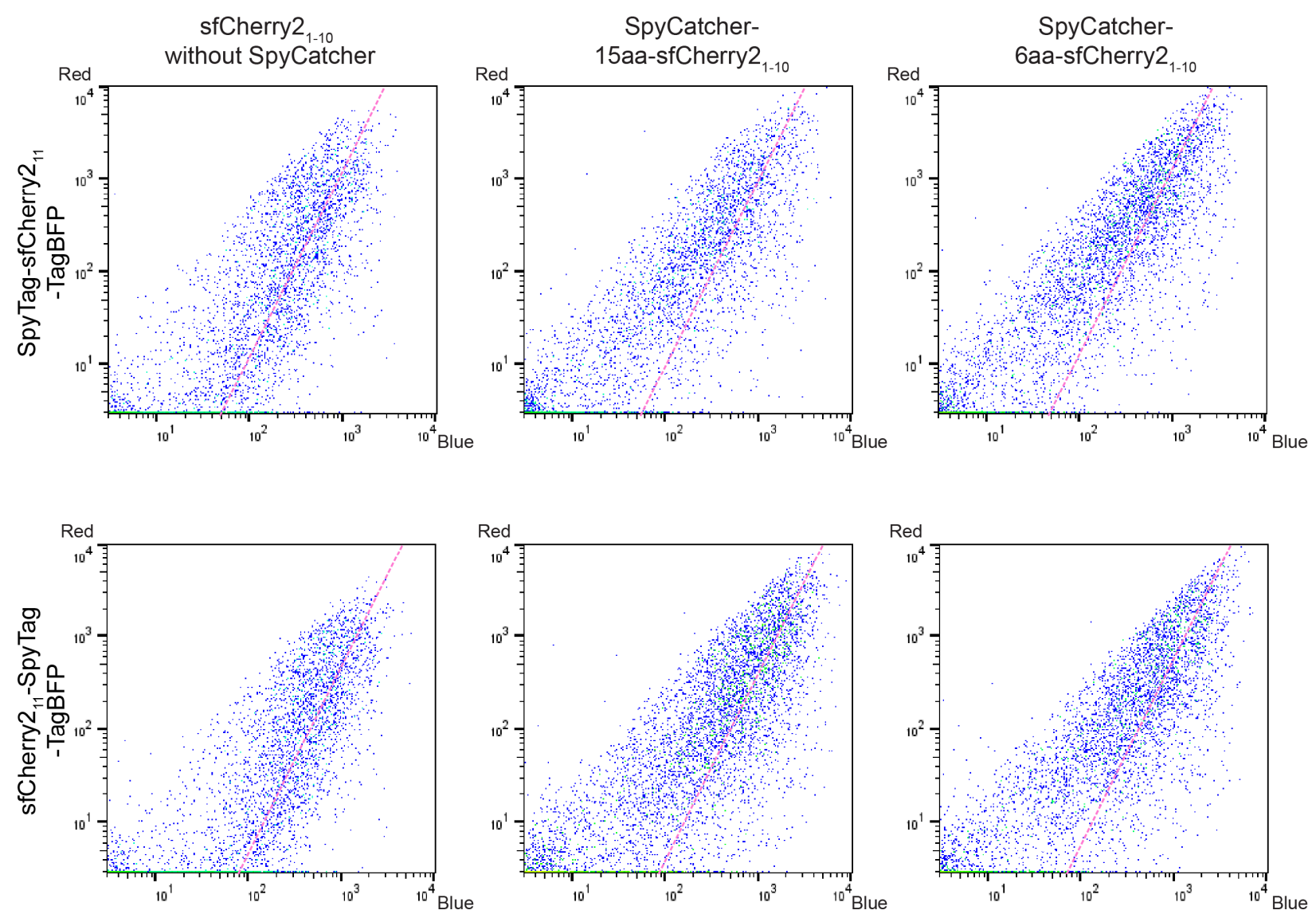

Supplementary Figure 2. Flow cytometry analysis of whole cell fluorescence in HEK 293T cells expressing different tandem tags (upper: SpyTag-sfCherry $2_{11}$-TagBFP, lower: sfCherry $2_{11}$ - SpyTagTagBFP) with different binders (left: sfCherry $2_{1-10}$ alone, middle: SpyCatcher-15aa-sfCherry $2_{1-10}$, right: SpyCatcher-6aa-sfCherry $2_{1-10}$ ). The pink dashed trend line has a slope of 2. 


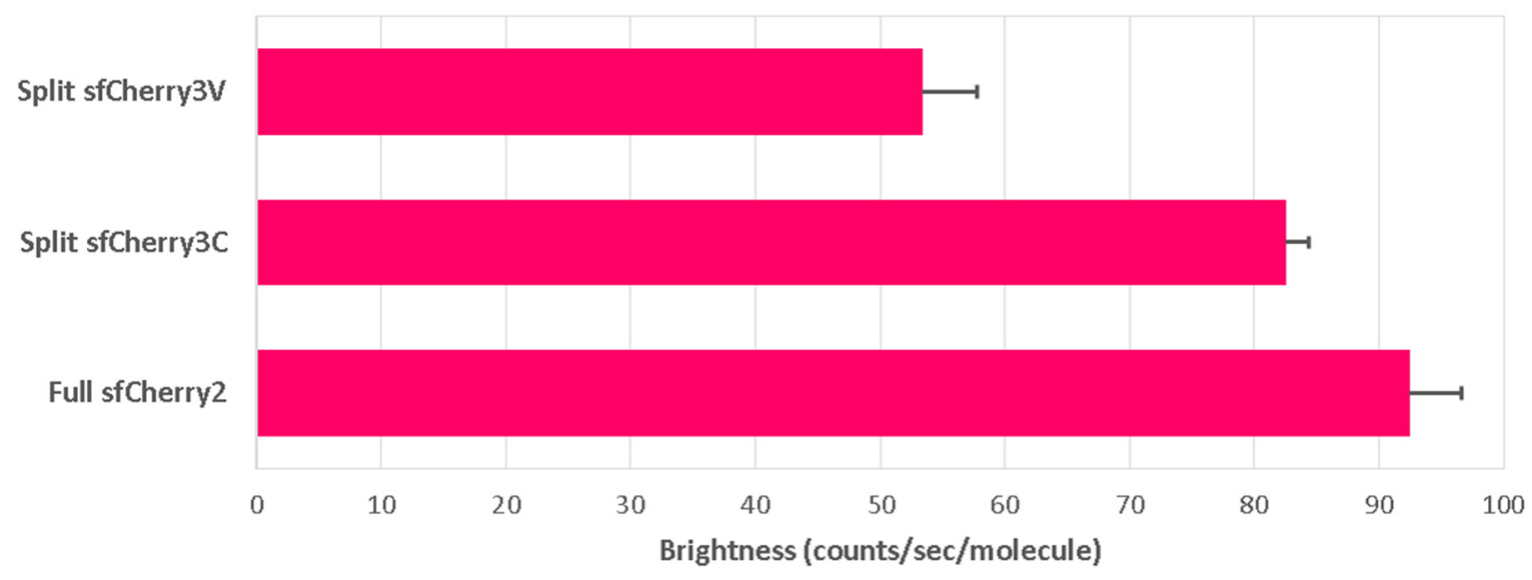

Supplementary Figure 3. Single-molecule brightness measurement of split sfCherry3V, split sfCherry3Cs and full-length sfCherry 2 using fluorescence fluctuation spectroscopy by two-photon excitation. 

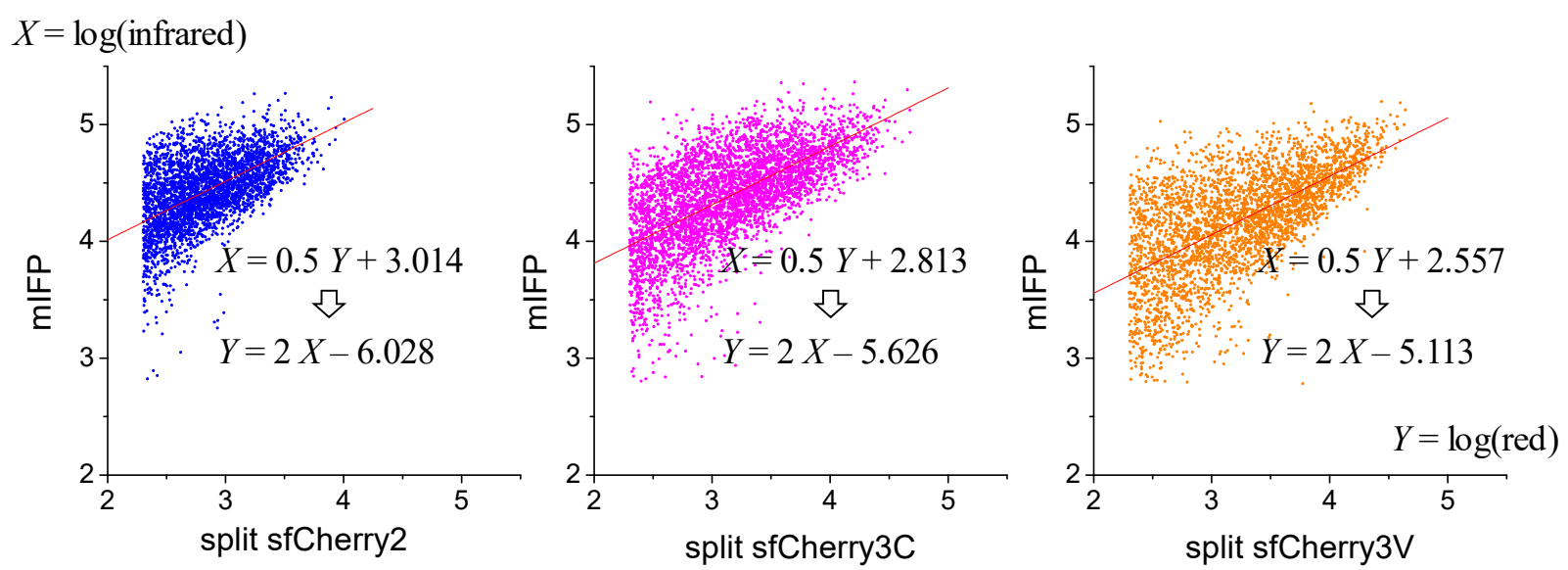

Supplementary Figure 4. Least square linear fitting of flow cytometry points in Figure 3E with a fixed slope of 0.5 . The $x$ - and $y$-axes were flipped compared to main figures in order to properly handle the asymmetric errors in the two channels. Points with intensities within $3 \sigma$ of the scattering background in either channel were excluded from the fitting. The intercept change in the fitted line (from $3.014 \pm 0.004$ to $2.813 \pm 0.005$ to $2.557 \pm 0.006, \pm$ standard error for least square fitting) was used to calculate the relative change in dissociation constant $K_{D}{ }^{\prime}$ (see Supplementary Notes). 


\section{Supplementary Notes}

\section{A two-step model for FP $_{1-10 / 11}$ complementation}

The overall complementation process of commonly used split fluorescent proteins is known to be irreversible $[1,2]$. Here, we verified that in the case of the self-complementing split mNG2 $2_{1-10 / 11}$, the overall process is also irreversible by fluorescence recovery after photobleaching (FRAP) experiment. In HEK $293 \mathrm{~T}$ cells with lamin A/C endogenously labeled by $\mathrm{mNG}_{11}$ and constitutively overexpressed mNG2 $2_{1-10}$ [3], bleaching a part of the nuclear lamina led to no observed fluorescence recovery in $10 \mathrm{~min}$ (Supplementary Figure 1, Supplementary Movie). Considering the fast maturation of mNeonGreen $(<10$ $\mathrm{min}$ ) [4], this lack of fluorescence recovery indicated that there was no exchange between the complemented mNG2 $2_{1-10}$ with free cytosolic mNG2 $2_{1-10}$.

On the other hand, a one-step, irreversible complementation would result in a direct proportional relationship between the complemented fluorescence signal and the fragment expression level. In this case, the FACS data points in Figure 1C (left) should follow a trend line with a slope of 1, whereas in reality, they fall closer to a trend line with a slope of 2 . To explain this observation, we consider a twostep complementation model, in which the first step is a relatively fast reversible binding between the $\mathrm{FP}_{1 \text { - }}$ 10 and $\mathrm{FP}_{11}$ fragments, forming an intermediate $\mathrm{FP}^{*}{ }_{1-10 / 11}$ complex, followed by a second, slower step of irreversible folding and/or fluorophore maturation.

$$
\mathrm{FP}_{1-10}+\mathrm{FP}_{11} \underset{\text { binding }}{\stackrel{K_{D}}{\rightleftharpoons}} \mathrm{FP}_{1-10 / 11}^{*} \underset{\text { folding/maturation }}{\stackrel{k_{f}}{\longrightarrow}} \mathrm{FP}_{1-10 / 11} \stackrel{k_{\text {deg }}}{\rightarrow} \text { degradation }
$$

At a steady state, the concentration of the complemented, functionally fluorescent species, $\mathrm{FP}_{1-10 / 11}$, is related to the concentration of the intermediate complex, $\mathrm{FP}^{*}{ }_{1-10 / 11}$, by:

$$
\left[\mathrm{FP}_{1-10 / 11}\right]=\frac{k_{f}}{k_{\text {deg }}}\left[\mathrm{FP}_{1-10 / 11}^{*}\right]
$$

where $k_{f}$ and $k_{d e g}$ are the folding/maturation rate and degradation rate, respectively. Generally, we would expect that the folding rate is much faster than the degradation rate, i.e. $k_{f}>>k_{d e g}$ and hence

$\left[\mathrm{FP}_{1-10 / 11}\right]>>\left[\mathrm{FP}_{1-10 / 11}^{*}\right]$. The concentration of $\mathrm{FP}^{*}{ }_{1-10 / 11}$ is further connected to the concentration of the free fragments as:

$$
\left[\mathrm{FP}_{1-10 / 11}^{*}\right]=\frac{1}{K_{D}}\left[\mathrm{FP}_{1-10}\right]\left[\mathrm{FP}_{11}\right]
$$

where $K_{D}$ is the dissociation constant for the initial binding step. In our experiment, $\mathrm{FP}_{11}$ and $\mathrm{FP}_{1-10}$ are expressed from co-transfected plasmids. Figure 1E indicates that their expression levels in individual cells follow a proportional relationship:

$$
\left[\mathrm{FP}_{1-10}\right]=a\left[\mathrm{FP}_{11}\right]
$$

where $a$ should be close to 1 considering that the same expression vectors are used for $\mathrm{FP}_{11}$ and $\mathrm{FP}_{1-10}$. Taken together, we have:

$$
\left[\mathrm{FP}_{1-10 / 11}\right]=\frac{1}{K_{D}{ }^{\prime}}\left[\mathrm{FP}_{11}\right]^{2}
$$


where the effective dissociation constant

$$
K_{D}{ }^{\prime}=\frac{k_{d e g}}{k_{f} a} K_{D}
$$

In our FACS experiment, the complemented fluorescence signal, $Y$, is proportional to [ $\left.\mathrm{FP}_{1-10 / 11}\right]$, whereas the expression marker (mIFP) signal, $X$, is actually proportional to the total concentration of complemented and uncomplemented $\mathrm{FP}_{11}$ fragment

$$
X \propto\left[\mathrm{FP}_{1-10 / 11}\right]+\left[\mathrm{FP}_{1-10 / 11}^{*}\right]+\left[\mathrm{FP}_{11}\right] \approx\left[\mathrm{FP}_{1-10 / 11}\right]+\left[\mathrm{FP}_{11}\right]=\left[\mathrm{FP}_{1-10 / 11}\right]+\sqrt{K_{D}{ }^{\prime}\left[\mathrm{FP}_{1-10 / 11}\right]}
$$

considering that $\left[\mathrm{FP}_{1-10 / 11}\right]>>\left[\mathrm{FP}_{1-10 / 11}^{*}\right]$.

The relationship between $Y$ and $X$, on a log-log plot, follows the black curve on the simulated plot below:

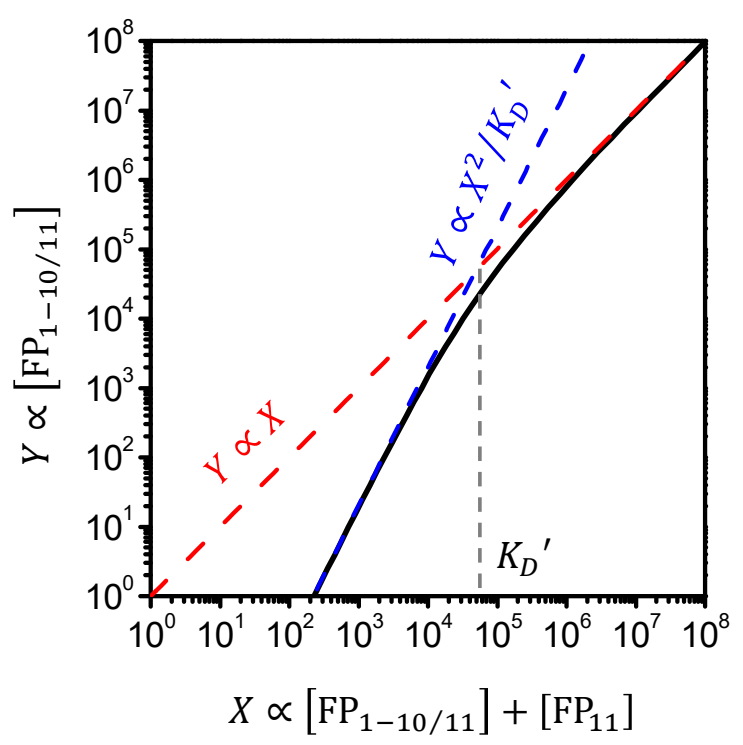

At a high fragment expression level or with a small $K_{D}{ }^{\prime}$ (the case of $\mathrm{GFP}_{1-10 / 11}$ ), $X$ is dominated by the first term of $\left[\mathrm{FP}_{1-10 / 11}\right]$, making the right end of the curve approaching a slope of 1 . On the other hand, at a low fragment expression level or with a large $K_{D}{ }^{\prime}$ (the cases of $\mathrm{mNG} 2_{1-10 / 11}$ and sfCherry $2_{1-10 / 11}$ ), $X$ is dominated by the second term of $\left[\mathrm{FP}_{11}\right]=\sqrt{K_{D}{ }^{\prime}\left[\mathrm{FP}_{1-10 / 11}\right]}$, making the left end of the curve approaching a slope of 2 .

In this latter regime, only a small fraction of the $\mathrm{FP}_{11}$ fragment is complemented with the $\mathrm{FP}_{1-10}$ fragment to reconstitute a functional fluorescent protein. Consequently, the apparent brightness of the $\mathrm{FP}_{11}$-labeled protein is lower than protein labeled by the full length FP. The complementation efficiency, defined as the fraction of $\mathrm{FP}_{11}$ complemented with $\mathrm{FP}_{1-10}$, is directly proportional to the concentration of $\mathrm{FP}_{1-10}$ :

$$
\text { complementation efficiency }=\frac{\left[\mathrm{FP}_{1-10 / 11}\right]}{\left[\mathrm{FP}_{1-10 / 11}\right]+\left[\mathrm{FP}_{1-10 / 11}^{*}\right]+\left[\mathrm{FP}_{11}\right]} \approx \frac{\left[\mathrm{FP}_{1-10 / 11}\right]}{\left[\mathrm{FP}_{11}\right]}=\frac{\left[\mathrm{FP}_{1-10}\right]}{K_{D} "}
$$


where $K_{D} "=a K_{D}{ }^{\prime}$ (i.e. effective dissociation constant with respect to $\mathrm{FP}_{1-10}$ instead of $\mathrm{FP}_{11}$ ). This relationship holds true both for our FACS experiment and for any general applications of $\mathrm{FP}_{11}$ labeling. In other words, the complementation efficiency improves with higher expression level of the $\mathrm{FP}_{1-10}$ fragment. Therefore, overexpression of $\mathrm{mNG} 2_{1-10}$ and sfCherry $2_{1-10}$ improves the apparent brightness of the $\mathrm{FP}_{11}$ labeled proteins. This improvement saturates when the concentration of $\mathrm{FP}_{1-10}$ is approaching $K_{D}{ }^{\prime \prime}$. Therefore, a trade-off must be considered between the diminishing brightness improvement and the potential cytotoxicity of high $\mathrm{FP}_{1-10}$ expression.

Obviously, a better approach to improve the apparent brightness / complementation efficiency is to reduce $K_{D}$ ' (equivalent to reducing $K_{D}{ }^{\prime \prime}$ ). This is exactly what we tried to achieve by engineering sfCherry3C and sfCherry3V. In these cases, the improvement of $K_{D}$ ' can be measured by fitting the FACS data with a line at a slope of 2 and then measure the change in the intercept (fig. S4).

From sfCherry2 to sfCherry3C, the $K_{D}{ }^{\prime}$ decreased by:

$$
\frac{K_{D}{ }^{\prime}(\text { sfCherry } 2)}{K_{D}{ }^{\prime}(\text { sfCherry3C })}=10^{6.028-5.628}=2.5
$$

From sfCherry2 to sfCherry $3 \mathrm{~V}$, the $K_{D}{ }^{\prime}$ decreased by:

$$
\frac{K_{D}{ }^{\prime}(\text { sfCherry } 2)}{K_{D}{ }^{\prime}(\text { sfCherry } 3 \mathrm{~V})}=10^{6.028-5.113}=8.2
$$

\section{References}

1. Cabantous, S. and G.S. Waldo, In vivo and in vitro protein solubility assays using split GFP. Nat Methods, 2006. 3(10): p. 845-54.

2. Kerppola, T.K., Visualization of molecular interactions by fluorescence complementation. Nat Rev Mol Cell Biol, 2006. 7(6): p. 449-56.

3. Feng, S., et al., Improved split fluorescent proteins for endogenous protein labeling. Nat Commun, 2017. 8(1): p. 370.

4. Shaner, N.C., et al., A bright monomeric green fluorescent protein derived from Branchiostoma lanceolatum. Nat Methods, 2013. 10(5): p. 407-9. 


\section{Supplementary Tables}

Supplementary Table 1: Sequence of sfCherry3C $C_{1-10}$, sfCherry3 $_{1-10}$, SpyCatcher 002 and SpyTag 002.

\begin{tabular}{|c|c|}
\hline & DNA Sequence \\
\hline sfCherry3C $C_{1-10}$ & $\begin{array}{l}\text { GAGGAGGACAACATGGCCATCATCAAGGAGTTCATGAGATTCAAGGTG } \\
\text { CACATGGAGGGCAGCGTGAACGGCCACGAGTTCGAGATCGAGGGCGA } \\
\text { GGGCGAGGGCCACCCCTACGGGGCACCCAGACCGCCAGGCTGAAGGT } \\
\text { GACCAAGGGCGACCCCCTGCCTTCGCCTGGGACATCCTGAGCCCCCA } \\
\text { GTTCATGTACGGCAGCAAGGCCTACGTGAAGCACCCCGCCGACATCCC } \\
\text { CGACTACCTGAAGCTGAGCTTCCCCGAGGGCTTCACCTGGGAGAGAGT } \\
\text { GATGAACTTCGAGGACGGCGGCGTGGTGGCCGTGACCCAGGACAGCAG } \\
\text { CCTGCAGGACGGCCAGTTCATCTACAAGGTGAAGCTGCTGGGCATCAA } \\
\text { CTTCCCCAGCGACGGCCCCGTGATGCAGAAGAAGACCATGGGCTGGGA } \\
\text { GGCCAGCACCGAGAGAATGTACCCCGAGGACGGCGCCCTGAAGGGCG } \\
\text { AGATCAACCAGAGACTGAAGCTGAAGGACGGCGGTCACTACGACGCCG } \\
\text { AGGTGAAGACCACCTACAGGGCCAAGAAGCCCGTGCAGCTGCCCGGCG } \\
\text { CCTACGACGTGGACATCAAGCTGGACATCACCAGCCACAACGAGGAC }\end{array}$ \\
\hline sfCherry3 $V_{1-10}$ & $\begin{array}{l}\text { GAGGAGGACAACATGGCCATCATCAAGGAGTTCATGAGATTCAAGGTG } \\
\text { CACATGGAGGGCAGCGTGAACGGCCACGAGTTCGAGATCGAGGGCGA } \\
\text { GGGCGAGGGCCACCCCTACGAGGGCACCCAGACCGCCAGGCTGAAGGT } \\
\text { GACCAAGGGCGACCCCCTGCCTTCGCCTGGGACATCCTGAGCCCCCA } \\
\text { GTTCATGTACGGCAGCAAGGCCTACGTGAAGCACCCCGCCGACATCCC } \\
\text { CGACTACTGGAAGCTGAGCTTCCCCGAGGGCTTCACCTGGGAGAGAGT } \\
\text { GATGAACTTCGAGGACGGCGGCGTGGTGGCCGTGACCCAGGACAGCAG } \\
\text { CCTGCAGGACGGCCAGTTCATCTACAAGGTGAAGCTGCTGGGCATCAA } \\
\text { CTTCCCCAGCGACGGCCCCGTGATGCAGAAGAAGACCATGGGCTGGGA } \\
\text { GGCCAGCACCGAGAGAATGTACCCCGAGGACGGCGCCCTGAAGGGCG } \\
\text { AGATCAACCAGAGACTGAAGCTGAAGGACGGCGGTCACTACGACGCCG } \\
\text { AGGTGAAGACCACCTACAGGGCCAAGAAGCCCGTGCAGCTGCCCGGCG } \\
\text { CCTACGACGTGGACATCAAGCTGGACATCACCAGCCACAACGAGGAC }\end{array}$ \\
\hline SpyCatcher 002 & $\begin{array}{l}\text { GGCGCCATGGTAACCACCTTATCAGGTTTATCAGGTGAGCAAGGTCCGT } \\
\text { CCGGTGATATGACAACTGAAGAAGATAGTGCTACCCATATTAAATTCTC } \\
\text { AAAACGTGATGAGGACGGCCGTGAGTTAGCTGGTGCAACTATGGAGTT } \\
\text { GCGTGATTCATCTGGTAAAACTATTAGTACATGGATTTCAGATGGACAT } \\
\text { GTGAAGGATTTCTACCTGTATCCAGGAAAATATACATTTGTCGAAACCG } \\
\text { CAGCACCAGACGGTTATGAGGTAGCAACTGCTATTACCTTTACAGTTAA } \\
\text { TGAGCAAGGTCAGGTTACTGTAAATGGCGAAGCAACTAAAGGTGACGC } \\
\text { TCATACT }\end{array}$ \\
\hline & GTGCCTACTATCGTGATGGTGGACGCCTACAAGCGTTACAAG \\
\hline
\end{tabular}


Supplementary Table 2: DNA sequence used for sgRNA in vitro transcription

\begin{tabular}{|l|l|l|}
\hline $\begin{array}{l}\text { Target } \\
\text { Gene }\end{array}$ & $\begin{array}{l}\text { Target } \\
\text { Term }\end{array}$ & Sequence of DNA oligo for sgRNA synthesis \\
\hline LMNA & $\mathrm{N}$ & $\begin{array}{l}\text { TAATACGACTCACTATAGGCCATGGAGACCCCGTCCCAGGTTTAAGA } \\
\text { GCTATGCTGGAA }\end{array}$ \\
\hline CLTA & $\mathrm{N}$ & $\begin{array}{l}\text { TAATACGACTCACTATAGGGCCATGGCGGGCAACTGAAGTTTAAGA } \\
\text { GCTATGCTGGAA }\end{array}$ \\
\hline RAB11A & $\mathrm{N}$ & $\begin{array}{l}\text { TAATACGACTCACTATAGGGTAGTCGTACTCGTCGTCGGTTTAAGAG } \\
\text { CTATGCTGGAA }\end{array}$ \\
\hline HP1b & $\mathrm{N}$ & $\begin{array}{l}\text { TAATACGACTCACTATAGGAAAGCTGGCGGGCACTATGGTTTAAGA } \\
\text { GCTATGCTGGAA }\end{array}$ \\
\hline SEC61b & $\mathrm{N}$ & $\begin{array}{l}\text { TAATACGACTCACTATAGGCTTGTCTCCCTCTACAGCCGTTTAAGAG } \\
\text { CTATGCTGGAA }\end{array}$ \\
\hline ARL6IP1 & $\mathrm{N}$ & $\begin{array}{l}\text { TAATACGACTCACTATAGGATCCCCGAGACGATGGCGGGTTTAAGA } \\
\text { GCTATGCTGGAA }\end{array}$ \\
\hline
\end{tabular}


Supplementary Table 3: Oligo-nucleotide donor DNA sequence

\begin{tabular}{|c|c|}
\hline Target Gene-FP 11 Tag & DNA sequence \\
\hline LMNA-sfCherry $2_{11}$ & $\begin{array}{l}\text { TCCTTCGACCCGAGCCCCGCGCCCTTTCCGGGACCCCTGCCCCGC } \\
\text { GGGCAGCGCTGCCAACCTGCCGGCCATGTACACCATCGTGGAGC } \\
\text { AGTACGAGAGAGCCGAGGCCAGACACAGCACCGGTGGCGGCGA } \\
\text { GACCCCGTCCCAGCGGCGCGCCACCCGCAGCGGGGCGCAGGCCA } \\
\text { GCTCCACTCCGCTGTCGCCCACCC }\end{array}$ \\
\hline CLTA-sfCherry 211 & $\begin{array}{l}\text { CGGGCGTGGTGTCGGTGGGTCGGTTGGTTTTTGTCTCACCGTTGG } \\
\text { TGTCCGTGCCGTTCAGTTGCCCGCCATGTACACCATCGTGGAGCA } \\
\text { GTACGAGAGAGCCGAGGCCAGACACAGCACCGGAGGTGGCATG } \\
\text { GCTGAGCTGGATCCGTTCGGCGCCCCTGCCGGCGCCCCTGGCGGT } \\
\text { CCCGCGCTGGGGAACGGAGTGG }\end{array}$ \\
\hline RAB11A-sfCherry $2_{11}$ & $\begin{array}{l}\text { CCCTGCAGCGACGCCCCCTGGTCCCACAGATACCACTGCTGCTCC } \\
\text { CGCCCTTTCGCTCCTCGGCCGCGCAATGTACACCATCGTGGAGCA } \\
\text { GTACGAGAGAGCCGAGGCCAGACACAGCACCGGAGGTGGCGGC } \\
\text { ACCCGCGACGACGAGTACGACTACCTCTTTAAAGGTGAGGCCAT } \\
\text { GGGCTCTCGCACTCTACACAGTC }\end{array}$ \\
\hline HP1b-sfCherry $2_{11}$ & $\begin{array}{l}\text { CTAATGCCCTTTTTATTTCATTTATCATTTTAGCAGCGTCACCCTT } \\
\text { TACACCAGAAAGCTGGCGGGCACTATGTACACCATCGTGGAGCA } \\
\text { GTACGAGAGAGCCGAGGCCAGACACAGCACCGGTGGCGGCGGG } \\
\text { AAAAAACAAAACAAGAAGAAAGTGGAGGAGGTGCTAGAAGAGG } \\
\text { AGGAAGAGGAATATGTGGTGGAAA }\end{array}$ \\
\hline SEC61b-sfCherry $2_{11}$ & $\begin{array}{l}\text { GTGTCTAGGCCGGGGTTCTGGGGCAGGCCTGCCGCGCTCACCCG } \\
\text { TCTGTCTGCTTGTCTCCCTCTACAGTACACCATCGTGGAGCAGTA } \\
\text { CGAGAGAGCCGAGGCCAGACACAGCACCGGTGGCGGCCCTGGTC } \\
\text { CGACCCCCAGTGGCACTAACGTGGGATCCTCAGGGCGCTCTCCC } \\
\text { AGCAAAGCAGTGGCCGCCCGGGC }\end{array}$ \\
\hline ARL6IP1-sfCherry $2_{11}$ & $\begin{array}{l}\text { GCGGGTTTCGGTTGGAGGACTCGTTGGGGAGGTGGCCTGCGCTT } \\
\text { GTAGAGACTGCATCCCCGAGACGATGTACACCATCGTGGAGCAG } \\
\text { TACGAGAGAGCCGAGGCCAGACACAGCACCGGTGGCGGCGCGG } \\
\text { AGGGAGATAATCGCAGCACCAACCTGCTGGTGAGTCCTGGCTGC } \\
\text { CTGTCCCCCGGGAGCCGAGCGA }\end{array}$ \\
\hline
\end{tabular}

sfCherry $2_{11}$ sequence

Linker sequence

Coding region sequence 